\title{
Inertioelastic Flow Instability at a Stagnation Point
}

\author{
Noa Burshtein, ${ }^{1}$ Konstantinos Zografos, ${ }^{2}$ Amy Q. Shen, ${ }^{1}$ Robert J. Poole, ${ }^{2}$ and Simon J. Haward ${ }^{1}$ \\ ${ }^{1}$ Okinawa Institute of Science and Technology Graduate University, Onna, Okinawa 904-0495, Japan \\ ${ }^{2}$ School of Engineering, University of Liverpool, Brownlow Street, Liverpool L69 3GH, United Kingdom \\ (Received 24 June 2017; revised manuscript received 8 September 2017; published 17 November 2017)
}

A number of important industrial applications exploit the ability of small quantities of high molecular weight polymer to suppress instabilities that arise in the equivalent flow of Newtonian fluids, a particular example being turbulent drag reduction. However, it can be extremely difficult to probe exactly how the polymer acts to, e.g., modify the streamwise near-wall eddies in a fully turbulent flow. Using a novel crossslot flow configuration, we exploit a flow instability in order to create and study a single steady-state streamwise vortex. By quantitative experiment, we show how the addition of small quantities (parts per million) of a flexible polymer to a Newtonian solvent dramatically affects both the onset conditions for this instability and the subsequent growth of the axial vorticity. Complementary numerical simulations with a finitely extensible nonlinear elastic dumbbell model show that these modifications are due to the growth of polymeric stress within specific regions of the flow domain. Our data fill a significant gap in the literature between the previously reported purely inertial and purely elastic flow regimes and provide a link between the two by showing how the instability mode is transformed as the fluid elasticity is varied. Our results and novel methods are relevant to understanding the mechanisms underlying industrial uses of weakly elastic fluids and also to understanding inertioelastic instabilities in more confined flows through channels with intersections and stagnation points.

DOI: 10.1103/PhysRevX.7.041039

Subject Areas: Fluid Dynamics, Nonlinear Dynamics, Soft Matter

\section{INTRODUCTION}

The addition of high molecular weight polymers to a simple Newtonian fluid at even extremely low concentrations (parts per million) imparts a small but important degree of elasticity to the liquid, which can have a dramatic effect on its macroscopic flow behavior. The effects of polymer additives are exploited for the reduction of drag in turbulent flows [1-6], for enhancing the pressure drop in porous media flows [7], and for inhibiting jet breakup and the atomization of sprays $[8,9]$. These effects are broadly understood in terms of the generation of localized anisotropic elastic stresses due to polymer stretching and orientation in specific regions of the flow field. The magnitude of the elastic stress (relative to the viscous stress) is characterized by the ratio between the deformation rate of the flow and the relaxation rate of the polymer, i.e., the Weissenberg number (Wi). However, detailed understanding of the mechanisms underlying the phenomena, such as how exactly polymer stretching feeds back on the flow field in order to suppress (or generate) instabilities, in many instances remains vague. Systems designed to model aspects of these flows, while avoiding their full complexity,

Published by the American Physical Society under the terms of the Creative Commons Attribution 4.0 International license. Further distribution of this work must maintain attribution to the author(s) and the published article's title, journal citation, and DOI. can play a pivotal role in filling the remaining knowledge gaps. Such an approach is routine for porous media, where cylinders or spheres can be used to model individual elements of a porous array [10-13]. Aspects of jet or spray breakup can likewise be approximated by capillary thinning or drop pinch-off experiments [14-17]. However, modeling an individual element of a turbulent flow (e.g., a vortex) in a way that can be easily studied in detail is much more challenging.

Here, we address this challenge by exploiting a recently reported symmetry-breaking flow bifurcation that occurs for Newtonian flow in the cross-slot geometry [Fig. 1(a)], which results in the formation of a single three-dimensional steady vortex, axially aligned along the outlet channels [18]. The cross-slot geometry is formed by mutually bisecting rectangular channels of constant width $w$ and depth $d$. When fluid is injected at equal rates into two opposing inlet channels of the cross slot, and is withdrawn at an equal and opposite rate from the two remaining outlets, the symmetry of the flow field under creeping-flow conditions results in the formation of a singular hyperbolic point (a stagnation point) at the location $x=y=0$. In the vicinity of the stagnation point the flow field approximates to pure planar elongation $[19,20]$. Instability occurs as the Reynolds number (Re) (which is the ratio of inertial to viscous forces) is increased above a critical value $\operatorname{Re}_{c} \approx 40$ (for $w=d$ ) [18]. The instability is initiated by random imbalances between four cells of Dean vortices that form in the cross section of the outlet channel, causing the 


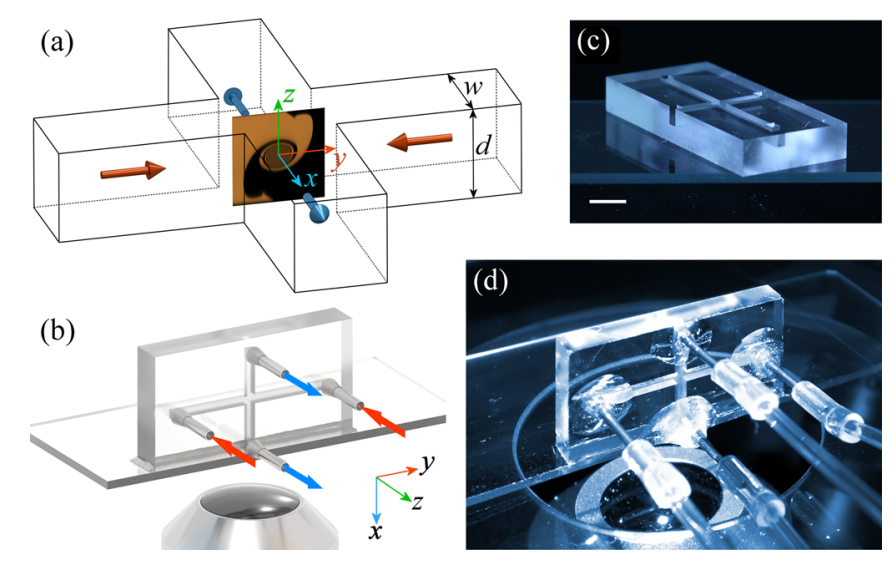

FIG. 1. The cross-slot geometry, where inflow is along the $y$ direction (indicated by the red arrows) and outflow is along the $x$ direction (indicated by the blue arrows). (a) Schematic diagram of a cross-slot device showing the characteristic channel dimensions and the coordinate system, with origin at the geometric center of the crossing channels. A spiral vortex forms along the $x$ axis for $\operatorname{Re}>\operatorname{Re}_{c}$. (b) A scheme illustrating a design for a microfluidic cross-slot device allowing direct observation of the $z-y$ plane at $x=0$. The device is vertically mounted on an inverted microscope with a long working distance lens, enabling a direct view of the stagnation point region at the center of the geometry where the spiral vortex instability forms. (c) A prototype microfluidic crossslot device for illustrative purposes (with channel dimensions $w=d=1 \mathrm{~mm}$ ), fabricated in fused silica using selective laserinduced etching: scale bar $5 \mathrm{~mm}$. (d) Photograph illustrating the experimental setup with the cross slot mounted on an inverted microscope.

introduction of an axial vorticity component at the stagnation point. Further vorticity growth is driven by vortex stretching in the elongational flow field [18] and steady state is reached when the vortex stretching becomes balanced by viscous diffusion [18,21]. The resulting system can then be considered as a steady-state proxy for an important aspect of a turbulent flow, namely, a streamwise stretched vortex [22-25]. Using a novel microfabrication technique, we reconfigure the cross-slot geometry in a unique way that allows us to quantitatively measure the axial vorticity in the cross section of the outlet channel for the first time [Figs. 1(b)-1(d)]. The device is then used to conduct the first direct observations and quantitative measurements of the effects of very small quantities of polymer additives on a single steady streamwise vortex.

Apart from $\mathrm{Wi}$ and $\mathrm{Re}$, a useful dimensionless parameter for characterizing polymeric flows is the elasticity number $(\mathrm{El} \approx \mathrm{Wi} / \mathrm{Re}$, which quantifies the ratio of elastic to inertial forces in the flow). For Newtonian flows, $\mathrm{El} \equiv 0$ and instabilities are "purely inertial," depending only on the magnitude of Re. Flows of highly elastic fluids $(\mathrm{El} \gg 1)$ present distinct "purely elastic" instabilites that can arise even for negligible inertia. Increasing evidence suggests the generality of these instabilities being driven by the accumulation of elastic stresses along curving streamlines
[26-29]. In the cross-slot device, purely elastic instability modes are manifested as two-dimensional flow asymmetries, which can be steady or time dependent, depending on particular fluid and geometric parameters [20,29-36]. At higher Wi, these purely elastic instabilites can develop into spatiotemporally disordered fluctuations described as "elastic turbulence" [37-40]. High elasticity numbers are characteristic of polymeric fluids with long relaxation times, i.e., those with viscous solvents, high molecular weights, and relatively high concentrations, at small geometric length scales. However, for flows of highly dilute polymer solutions in low viscosity solvents in larger length scale flow configurations, typical of drag-reduced flows, inertia is not negligible $(0<\mathrm{El} \lesssim 1)$ and the flow can be termed "inertioelastic" (or equivalently "elastoinertial"). In this case there is a complex interaction and competition between inertial and elastic effects that remains to be fully described and understood.

For flow in a macroscale pipe, for which the transition to sustained turbulence occurred at $\operatorname{Re} \approx 2000$ in the Newtonian case, Samanta et al. showed that the transition became delayed to progressively higher Re as the polymer concentration was increased [4]. However, they observed a new type of turbulent fluctuation termed elastoinertial turbulence (EIT) due to the onset of an elastic instability driven by polymeric stresses (though for non-negligible inertia), for which the onset Re decreased significantly as the polymer concentration increased. As Re was increased beyond the onset of the instability, the measured friction factor directly approached the maximum drag-reduction asymptote [2], without any excursion towards the Blasius friction factor scaling expected for Newtonian turbulence. Furthermore, numerical simulations of the flow structures and dynamics showed that as the maximum drag-reduction asymptote was approached, streamwise-oriented vortices characteristic of Newtonian turbulence were suppressed.

By using our cross-slot-based flow system to make direct measurements of the axial vorticity as we systematically vary both $\mathrm{Re}$ and $\mathrm{El}$, we shed insights on possible mechanisms for the vortex suppression observed in the drag-reduced state of EIT at the scale of a single streamwise vortex. We manipulate El via control of the polymer concentration and the solvent viscosity, and by characterizing our model fluids in this way, rather than simply using polymer concentration, we are able to collapse data for the critical $\mathrm{Re}$ and Wi conditions in a highly generalized dimensionless form. Complementary numerical simulations using a finitely extensible nonlinear elastic (FENE) dumbbell model provide near-quantitative agreement with the experiments and furnish vital additional information not available from the experiment on the spatial distribution of elastic stress due to polymer stretching in the complex 3D flow field. We are thus able to strengthen the links between, and suggest clear potential mechanisms for, the onset of inertioelastic instabilities and the suppression of streamwise vorticity observed in drag-reduced flows. 
We note that apart from having potential to model particular aspects of turbulence, cross-slot flows are also of significant fundamental interest and practical importance in their own right. The device has been instrumental to furthering the understanding of polymer dynamics in strong flows [41-45], and as a consequence has become widely considered as a benchmark flow for the study of complex fluids. The device is also a component of central importance in a range of both fundamental and applied microfluidic technologies [19,46-52]. To ensure that such experiments are conducted under well-defined conditions, it is clearly of great importance to establish stability criteria for cross-slot flows. Our new results fill a significant gap in the literature between the purely inertial [18] and purely elastic regimes (e.g., Ref. [29]) and link the two by showing how the instability is transformed from an inertia-dominated to an elasticity-dominated mode as $\mathrm{El}$ is increased. The crossslot device is considered a canonical planar intersecting geometry with a free stagnation point and we expect our results to have a wide relevance to more general flows of weakly elastic fluids in geometries characterized by colliding fluid streams and internal stagnation points (e.g., Refs. [5357]). Further, the experimental methods we present are readily transferable to the study of $3 \mathrm{D}$ and time-dependent flows and vortex dynamics in other geometries apart from the cross slot, and so are expected to have wide interest and applicability.

\section{METHODS}

\section{A. Device fabrication}

A microfluidic cross-slot device, with dimensions of $d=w=440 \pm 5 \mu \mathrm{m}$ and an aspect ratio $\alpha=w / d=1$, was fabricated using the state-of-the-art "LightFab" 3D printer (LightFab GmbH, Germany), which utilizes the technique of selective laser-induced etching [58]. Laser writing is performed in bulk fused silica followed by chemical etching in $\mathrm{KOH}\left(\right.$ at $80^{\circ} \mathrm{C}$, in an ultrasonic bath). The laser-modified glass is etched at a rate of $100 \mu \mathrm{m} / \mathrm{h}$, which is 1000 times faster than the etching rate of the unmodified glass. The glass device [Fig. 1(c)] is made within one piece of transparent, nonporous, nonflexible substrate with high resolution $[\sim O(1 \mu \mathrm{m})]$ and can endure the use of organic solvents, high pressures, and high temperatures, if required. The device inlet lengths are set to be $13 \mathrm{~mm}$, giving a high ratio $(\approx 30)$ between the inlet length and the channel width in order to allow flow to become fully developed before the fluid arrives at the center of the cross-slot geometry. The outlets are designed to be as long as possible $(6 \mathrm{~mm})$, while still allowing imaging to be performed in the $y-z$ center plane (at $x=0$ ) with a long working distance microscope objective.

Flow within the glass microfluidic device is driven using four individually controlled servo-driven neMESYS syringe pumps (Cetoni GmbH, Germany) fitted with Hamilton
Gastight syringes and operating at a minimum of $10 \times$ (and typically $>50 \times$ ) the specified lowest "pulsation-free" dosing rate. Two of the pumps drive fluid into the two opposed inlets, while the other two pumps withdraw fluid simultaneously from the two outlets of the device (all at an equal volumetric flow rate $Q$ ). In order to minimize system compliance, connections between the syringes and the fluidic device are made with rigid polyethylene tubing.

\section{B. Flow field measurements}

Quantitative measurements of the flow field are made in the $y$ - $z$ center plane ( $x=0$ plane) of the cross-slot device (Fig. 1) using a microparticle image velocimetry ( $\mu$-PIV) system (TSI Inc., MN) [59,60]. For this purpose, test fluids are seeded with fluorescent particles (PS-FluoRed, MicroParticles $\mathrm{GmbH}$, Germany) of diameter $d_{p}=$ $2.08 \mu \mathrm{m}$ with excitation and emission wavelengths of 530 and $607 \mathrm{~nm}$, respectively. The microfluidic device is mounted on the stage of an inverted microscope (Nikon Eclipse $\mathrm{T} i$ ), and the $x=0$ plane is brought into focus using a long working distance lens (Nikon Plan Fluor, 10×, $\mathrm{NA}=0.3$, Working Distance $=16 \mathrm{~mm}$ ); see Fig. $1(\mathrm{~d})$. With this combination of particle size and objective lens, the measurement depth over which particles contribute to the determination of the velocity field is $\delta x_{m} \approx 40 \mu \mathrm{m}$ $\left(\delta x_{m}<0.1 w\right)$ [59].

The $\mu$-PIV system is equipped with a $1280 \times 800$ pixel high-speed CMOS camera (Phantom MIRO), which operates in frame-straddling mode and is synchronized with a dual-pulsed Nd:YLF laser light source with a wavelength of $527 \mathrm{~nm}$ (Terra PIV, Continuum Inc., CA). The laser illuminates the fluid with pulses of duration $\delta t<10 \mathrm{~ns}$, thus exciting the fluorescent particles, which emit at a longer wavelength. Reflected laser light is filtered out by a G-2A epifluorescent filter so that only the light emitted by the fluorescing particles is detected by the CMOS imaging sensor array. Images are captured in pairs (one image for each laser pulse), where the time between pulses $\Delta t$ is set by the instrument operator such that the average particle displacement between the two images in each pair is around 4 pixels. Image pairs are binned into interrogation areas of $32 \times 32$ pixels and cross-correlated using a standard $\mu$-PIV algorithm (TSI Inc.) to obtain velocity vectors spaced on a square grid of $12.8 \times 12.8 \mu \mathrm{m}$ in $y$ and $z$. The typical error on individual velocity vector components is around $\pm 0.05 U$, where $U=Q / w d$ is the average flow velocity in the channel.

\section{Experimental protocol}

Initially, in order to identify the approximate value of $\operatorname{Re}_{c}$ for each test fluid, several images are captured for fixed Re applied below and above the transition. Next, more detailed quasistatic experiments are conducted over a range of Reynolds numbers spanning $\operatorname{Re}<\operatorname{Re}_{c}$ to $\operatorname{Re}>\operatorname{Re}_{c}$ by programing the syringe pumps to perform ramps up and 
down in Re with small step increases or decreases of $0.1 \leq \operatorname{Re} \leq 0.4$, depending on the precise fluid system under study. Typically $5 \mathrm{~s}$ of steady flow is imposed at each increment in Re. We point out that this is much longer than the diffusion time scale of the least viscous sample (water) tested in our device, which is given by $t_{d}=w^{2} / \nu \approx 0.2 \mathrm{~s}$. We have also carried out dynamic experiments for both step increases and step decreases in Re, showing that steadystate conditions are achieved in either case within approximately $0.5 \mathrm{~s}$ (results not shown here). All experiments are carried out at $25 \pm 1{ }^{\circ} \mathrm{C}$.

Image pairs are captured at a rate of $5 \mathrm{~Hz}$ using the $\mu$-PIV system, typically yielding 25 velocity vector fields per step in Re. Avoiding data captured during the transient at the start of each Re step, velocity fields from each step are averaged using the software Tecplot Focus (Tecplot Inc., WA) and further processed to obtain streamline projections on the $x=0$ plane and the axial ( $x$ component) of the vorticity, $\omega_{x}=\left(\partial v_{z} / \partial y\right)-\left(\partial v_{y} / \partial z\right)$, where $v_{z}$ and $v_{y}$ are the $z$ and $y$ components of the local flow velocity vectors, respectively.

It is worthwhile to mention that for each test fluid the range of $\mathrm{Re}$ we can examine in this way is restricted fundamentally by limitations of the $\mu$-PIV system, which does not allow the value of $\Delta t$ to be varied during a given experiment. Given a fixed $\Delta t$, if the flow velocity (or $\mathrm{Re}$ ) during an experiment becomes too low, particles are displaced insufficiently in the time between laser pulses to obtain reliable vectors. Conversely, if the flow velocity becomes too high, then too many particles can become displaced into neighboring interrogation areas between laser pulses and the cross-correlation algorithm again fails to resolve vectors. Ideally, for optimal function of the algorithm, the particle displacement between laser pulses should be around 4 pixels. In our experiment $\Delta t$ must therefore be carefully selected in order that velocity fields can be resolved reliably over a range of flow rates that spans the onset of the flow instability.

\section{Materials}

The effects of increasing elasticity on the onset and development of flow instability in the cross-slot device are studied using solutions of a high molecular weight poly (ethyleneoxide) (PEO) $\left(M_{w}=4 \mathrm{MDa}\right.$, Sigma Aldrich) over a range of concentrations $0.0001 \leq c \leq 0.3$ wt\% (i.e., $1 \leq$ $c \leq 3000$ parts per million by weight). Solutions are prepared in two different solvents: deionized water and an aqueous solution of $8 \mathrm{wt} \%$ of a low molecular weight poly (ethyleneglycol) (PEG) $\left(M_{w}=8 \mathrm{kDa}\right.$, Sigma Aldrich). At this molecular weight, 8 wt \% aqueous PEG behaves as a Newtonian fluid with a viscosity approximately 4 times greater than water [61]. Stock PEO solutions are prepared by dispersing a weighed quantity of the polymer powder in the appropriate quantity of solvent. To assist dissolution while avoiding mechanical degradation of the PEO, only lowspeed (30 rpm) magnetic stirring is applied. Dissolution is allowed to proceed until no refractive index variations or residual gels are visible in the fluid (typically 24-48 h). Subsequently, the fluids are stored at $4{ }^{\circ} \mathrm{C}$ and are used within a maximum of 4 weeks. More dilute solutions are prepared by dilution of the stock fluids in the appropriate quantity of solvent.

\section{E. Test fluid characterization}

Steady flow curves of shear viscosity $\eta$ as a function of the imposed shear rate $\dot{\gamma}$ are measured for the polymer solutions and the solvents using a stress controlled rotational rheometer (Anton Paar, MCR 502) with a 50-mm diameter $1^{\circ}$ cone-and-plate fixture. The results are presented in Fig. 2. Most of the fluids are essentially nonshear-thinning over the imposed range of shear rates; however, for the two highest concentrations of PEO in water a mild reduction in viscosity is observed as the shear rate increases. To estimate the viscosity of these shearthinning fluids at arbitrary shear rates imposed within the microfluidic channel, the shear-thinning flow curves are fitted with a Carreau-Yasuda generalized Newtonian fluid (GNF) model [62]:

$$
\eta=\eta_{\infty}+\frac{\eta_{0}-\eta_{\infty}}{\left[1+\left(\dot{\gamma} / \dot{\gamma}^{*}\right)^{a}\right]^{(1-n) / a}}
$$

where $\eta_{0}$ and $\eta_{\infty}$ are the zero and infinite shear rate viscosities, $\dot{\gamma}^{*}$ is the characteristic shear rate for the onset of shear thinning, $n$ is the power-law index in the shearthinning region, and $a$ is a parameter that controls the sharpness of the transition to the power-law regime.

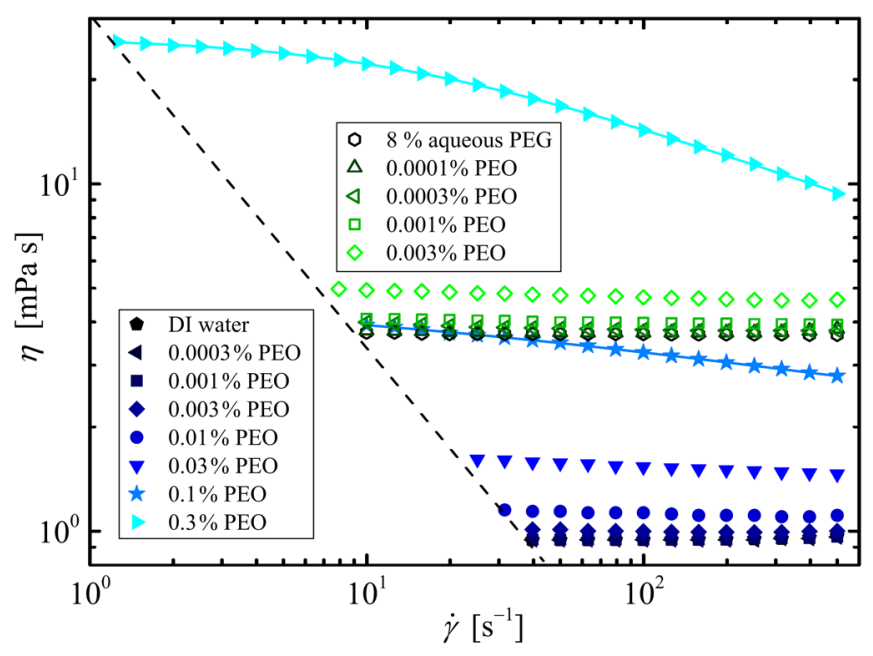

FIG. 2. Shear viscosity measurements with a stress-controlled rotational rheometer (Anton Paar MCR 502) for PEO solutions of various concentrations in two different solvents: water and $8 \mathrm{wt} \%$ aqueous PEG. Curve fitting for the shear-thinning fluids is done with the Carreau-Yasuda GNF model [Eq. (1)]. The diagonal dashed line represents $10 \times$ the minimum sensitivity of the rheometer. 
TABLE I. Parameters extracted by fitting the Carreau-Yasuda GNF model [Eq. (1)] to the steady flow curves of the shearthinning solutions of PEO dissolved in water.

\begin{tabular}{lccccr}
\hline \hline$c[\mathrm{wt} \%]$ & $\eta_{0}[\mathrm{mPas}]$ & $\eta_{\infty}[\mathrm{mPa} \mathrm{s}]$ & $\dot{\gamma}^{*}\left[\mathrm{~s}^{-1}\right]$ & $n$ & $a$ \\
\hline 0.1 & 3.9 & 1 & 22.7 & 0.83 & 1 \\
0.3 & 25 & 1 & 12.1 & 0.71 & 1 \\
\hline \hline
\end{tabular}

The values of these parameters for the two shear-thinning fluids are provided in Table I.

As far as possible, the relaxation times $\lambda$ of the test fluids are measured using capillary breakup extensional rheometry (CaBER, Thermo Scientific). For many of the more dilute PEO solutions, the relaxation times are too low to be measured accurately using the standard CaBER technique and therefore the more sensitive slow retraction method (SRM) is employed [63]. Two of the fluids (0.001 wt \% PEO in water and $0.0003 \mathrm{wt} \%$ PEO in PEG) are measured using both techniques and we obtain good agreement. For the lowest concentration $(0.0003 \mathrm{wt} \%)$, PEO in water, $\lambda$ could not be reliably measured even by the SRM and therefore we estimate the relaxation time of this fluid using the Zimm formula:

$$
\lambda_{\text {Zimm }}=F \frac{[\eta] M_{w} \eta_{s}}{N_{A} k_{B} T},
$$

where $M_{w}=4 \times 10^{6} \mathrm{~g} \mathrm{~mol}^{-1}$ is the quoted PEO molecular weight, $N_{A}$ is the Avogadro constant, $k_{B}$ is the Boltzmann constant, $T$ is the absolute temperature, $\eta_{s}=0.94 \mathrm{mPas}$ is the measured solvent viscosity, and the front factor $F=0.463$ [64]. The intrinsic viscosity $[\eta]=1.323 \mathrm{~m}^{3} \mathrm{~kg}^{-1}$ is calculated according to the MarkHouwink-Sakurada correlation [65]. According to this calculation the longest relaxation time of the PEO molecule in water is $\lambda_{\text {Zimm }} \approx 1 \mathrm{~ms}$.

The characteristic relaxation times of all the test fluids are summarized in Table II, along with their measured zero shear viscosities and the "solvent-to-total viscosity ratios" $\beta=\eta_{s} / \eta_{0}$, where $\eta_{0}=\eta_{s}+\eta_{p}$ and $\eta_{p}$ is the polymer contribution to the viscosity.

It is apparent by inspection of Fig. 2 and Table II that by using the more viscous aqueous solvent containing $8 \mathrm{wt} \%$ PEG, we are able to increase the relaxation time of the fluid while maintaining the viscosity ratio close to 1 and avoiding significant shear-thinning effects, which occur for higher concentrations of PEO in water.

Also shown in Table II is the ratio of the polymer concentration $c$ to the overlap concentration $c^{*}$. The overlap concentration for PEO in the aqueous-based solvents used here is estimated based on space filling of polymer coils with a radius of gyration $R_{g}$ [66]:

$$
c^{*}=\frac{3 M_{w}}{4 N_{A} \pi R_{g}^{3}},
$$

where the value of $R_{g}=152 \mathrm{~nm}$ is estimated according to previous results of light scattering experiments [67]. Equation (3) provides a value of $c^{*} \approx 0.045 \mathrm{wt} \%$.

The equilibrium root-mean-square end-to-end separation of the PEO molecule is given by $\left\langle r_{0}{ }^{2}\right\rangle^{1 / 2}=\sqrt{6} R_{g}=$ $372 \mathrm{~nm}$. The contour length may be estimated by $L_{c}=l M_{w} / m=25.4 \mu \mathrm{m}$, where $l=0.28 \mathrm{~nm}$ and $m=$ $44 \mathrm{Da}$ are the length and mass of the PEO repeat unit, respectively [68]. The ratio $L=L_{c} /\left\langle r_{0}{ }^{2}\right\rangle^{1 / 2} \approx 70$ indicates the highly extensible nature of this high molecular weight PEO sample. This estimate of $L$ is used to compute an appropriate extensibility parameter $\left(L^{2}=5000\right)$ used for subsequent numerical simulations; see Sec. II H.

\section{F. Dimensionless numbers}

Here, we define the dimensionless numbers that are used to characterize the inertial, viscous, and elastic forces throughout both the experiments and numerical simulations. The Reynolds number $\mathrm{Re}$ describes the ratio of inertial to viscous forces in the flow and is defined as

$$
\operatorname{Re}=\frac{\rho U w}{\eta(\dot{\gamma})},
$$

where $\eta(\dot{\gamma})$ is the shear-rate-dependent shear viscosity and $\rho$ is the fluid (solvent) density.

The Weissenberg number describes the relative importance of elastic to viscous forces in the flow and (in the absence of a solvent viscosity) can be expressed as $\mathrm{Wi}=N_{1} / \tau_{x y}=\lambda \dot{\gamma}$. Here, $N_{1}=\left(\tau_{x x}-\tau_{y y}\right)$ is the first normal stress difference, $\tau_{x y}$ is the shear stress, and $\dot{\gamma}=$ $U / w$ is the characteristic shear rate within the flow channel [69]. In this work we use an "effective" Weissenberg number $\mathrm{Wi}_{\text {eff }}$, which is scaled using the solvent-to-total viscosity ratio $\beta$ in order to account for the solvent contribution to $\tau_{x y}$, but not to $N_{1}$ :

$$
\mathrm{Wi}_{\mathrm{eff}}=\lambda \dot{\gamma}(1-\beta) .
$$

The elasticity number is given by the ratio between $\mathrm{Wi}_{\text {eff }}$ and $\mathrm{Re}$ and therefore describes the relative importance of elastic to inertial forces:

$$
\mathrm{El}=\frac{\lambda \eta(\dot{\gamma})}{\rho w^{2}}(1-\beta)
$$

For constant viscosity fluids (such as the majority of our test solutions and the simulations), $\mathrm{El}$ is independent of the applied shear rate. Values of El for our test fluids in the microfluidic cross-slot device are provided in Table II.

\section{G. Data analysis using the Landau model}

Velocity fields obtained in the $x=0$ plane of the crossslot device using $\mu$-PIV are used to locally evaluate the 
TABLE II. Composition and material parameters of the experimental test fluids at $25^{\circ} \mathrm{C}$.

\begin{tabular}{llllcrr}
\hline \hline Solvent & $c[\mathrm{wt} \%]$ & $c / c^{*}$ & $\eta_{0}[\mathrm{mPas}]$ & $\lambda[\mathrm{ms}]$ & \multicolumn{1}{c}{$\beta=\eta_{s} / \eta_{0}$} & $\mathrm{El}$ \\
\hline Water & 0.0003 & 0.007 & $0.95 \pm 0.01$ & $1.0^{\mathrm{a}}$ & $0.99 \pm 0.01$ & $(5 \pm 5) \times 10^{-5}$ \\
$\eta_{s}=(0.94 \pm 0.01) \mathrm{mPa} \mathrm{s}$ & 0.001 & 0.02 & $0.95 \pm 0.01$ & $3.6 \pm 0.3^{\mathrm{b}}$ & $0.99 \pm 0.01$ & $(1.8 \pm 1.8) \times 10^{-4}$ \\
$\rho=997 \mathrm{~kg} \mathrm{~m}^{-3}$ & 0.003 & 0.07 & $1.02 \pm 0.01$ & $5.0 \pm 0.4^{\mathrm{b}}$ & $0.92 \pm 0.01$ & $(2.1 \pm 0.3) \times 10^{-3}$ \\
$\beta=1$ & 0.01 & 0.22 & $1.12 \pm 0.02$ & $12 \pm 1^{\mathrm{c}}$ & $0.84 \pm 0.02$ & $(1.1 \pm 0.2) \times 10^{-2}$ \\
& 0.03 & 0.66 & $1.59 \pm 0.02$ & $23 \pm 1$ & $0.59 \pm 0.01$ & $(7.8 \pm 0.4) \times 10^{-2}$ \\
& 0.1 & 2.2 & $3.90 \pm 0.05$ & $44 \pm 2$ & $0.24 \pm 0.00$ & $0.68 \pm 0.03^{\mathrm{d}}$ \\
\hline 8 wt $\%$ PEG & 0.3 & 6.6 & $25.0 \pm 0.1$ & $84 \pm 4$ & $0.04 \pm 0.00$ & $10.4 \pm 0.5^{\mathrm{d}}$ \\
$\eta_{s}=(3.71 \pm 0.01) \mathrm{mPa} \mathrm{s}$ & 0.0001 & 0.002 & $3.81 \pm 0.02$ & $2.2 \pm 0.3^{\mathrm{b}}$ & $0.97 \pm 0.01$ & $(8.3 \pm 0.4) \times 10^{-4}$ \\
$\rho=1011 \mathrm{~kg} \mathrm{~m}^{-3}$ & 0.0003 & 0.007 & $3.92 \pm 0.05$ & $12 \pm 1^{\mathrm{c}}$ & $0.95 \pm 0.01$ & $(1.2 \pm 0.5) \times 10^{-2}$ \\
$\beta=1$ & 0.001 & 0.02 & $4.07 \pm 0.02$ & $28 \pm 1$ & $0.91 \pm 0.01$ & $(5.2 \pm 0.6) \times 10^{-2}$ \\
\hline \hline
\end{tabular}

${ }^{\mathrm{a}}$ Relaxation time estimated from the Zimm formula [Eq. (2)].

${ }^{b}$ Relaxation time measured using the slow retraction method (SRM) implemented on a capillary breakup extensional rheometer (CaBER).

${ }^{\mathrm{c}}$ Relaxation time measured using both standard CaBER and SRM techniques.

${ }^{\mathrm{d}}$ Representative El for the fluid calculated based on the zero shear viscosity $\eta_{0}$.

vorticity in the axial direction $\omega_{x}=\left(\partial v_{z} / \partial y\right)-\left(\partial v_{y} / \partial z\right)$. Although other possible parameters can be considered [18], in this work we use the measurement of an increase in the value of the center point axial vorticity $\left.\omega_{x}\right|_{x=y=z=0}$ as an indication of the onset of the inertia-dominated mode of instability. We define a dimensionless order parameter $\psi$ for the instability as follows:

$$
\psi=\left.\frac{w}{U} \omega_{x}\right|_{x=y=z=0} .
$$

For low $\operatorname{Re}, \psi=0$, but, as $\operatorname{Re}$ is increased above $\operatorname{Re}_{c}$, the value of $\psi$ can bifurcate to one of two stable solutions (positive or negative) that are expressed by the handedness of the spiral vortex that is formed. For Newtonian fluids, this bifurcation can be described using a sixth-order Landau-type polynomial potential minimized in the following form [18]:

$$
\varepsilon=\frac{\operatorname{Re}-\operatorname{Re}_{c}}{\operatorname{Re}_{c}}=k \psi^{4}+g \psi^{2}-h \psi^{-1} .
$$

Here, $\varepsilon$ is a normalized control parameter and the coefficients $g$ and $k$ determine the shape and order of the transition. When $g>0$, the forward bifurcation is analogous to a second-order phase transition. When $g=0$, the bifurcation at $\varepsilon=0$ is tricritical, and for $g<0$, the bifurcation turns backwards, analogous to a first-order hysteretic phase transition. The asymmetric term with coefficient $h$ accounts for system imperfections that lead to the selection of a favored handedness of the spiral. In our particular microfluidic cross-slot device, we believe slight wall imperfections are responsible for spirals to form with a favored positive vorticity (i.e., anticlockwise rotation in our reference frame). In the numerical simulations the handedness of the spiral is equally likely to occur in either direction (i.e., the bifurcation is perfect).

\section{H. Numerical method}

The numerical investigation of the inertially driven flow instability is achieved by performing three-dimensional computational fluid dynamics simulations based on the finite-volume technique [70]. The flow is considered to be laminar, incompressible, and isothermal and is governed by the equations of mass conservation and momentum:

$$
\begin{aligned}
\nabla \cdot \mathbf{u} & =\mathbf{0}, \\
\rho\left(\frac{\partial \mathbf{u}}{\partial t}+\mathbf{u} \cdot \nabla \mathbf{u}\right) & =-\nabla p+\nabla \cdot \boldsymbol{\tau},
\end{aligned}
$$

where $\mathbf{u}$ is the velocity vector, $p$ is the pressure, and $\boldsymbol{\tau}$ is the stress tensor which contains the solvent $\boldsymbol{\tau}_{s}$ and polymer $\boldsymbol{\tau}_{p}$ contributions. Therefore, the stress tensor in the momentum equation is defined as $\boldsymbol{\tau}=\boldsymbol{\tau}_{s}+\boldsymbol{\tau}_{p}$, where the solvent component is given by $\boldsymbol{\tau}_{s}=\eta_{s}\left(\nabla \mathbf{u}+\nabla \mathbf{u}^{\mathrm{T}}\right)$. To account for the effects of elasticity, the set of governing equations is completed with an appropriate constitutive equation for $\boldsymbol{\tau}_{p}$. Here, the modified Chilcott-Rallison model, FENE-MCR, is employed [71,72]:

$$
\boldsymbol{\tau}_{p}+\frac{\lambda}{g\left(\tau_{p}\right)} \stackrel{\nabla}{\boldsymbol{\tau}}_{p}=\eta_{p}\left(\nabla \mathbf{u}+\nabla \mathbf{u}^{\mathrm{T}}\right),
$$

where $\stackrel{\nabla}{\boldsymbol{\tau}}_{p}$ is the upper-convected derivative of the polymeric component of the stress tensor and $g\left(\tau_{p}\right)$ is a function of the stress tensor, defined as

$$
g\left(\tau_{p}\right)=\frac{L^{2}+\left(\lambda / \eta_{p}\right) \operatorname{Tr}\left(\boldsymbol{\tau}_{p}\right)}{L^{2}-3} .
$$


In the above equation, $\operatorname{Tr}\left(\boldsymbol{\tau}_{p}\right)$ refers to the trace operator of the polymeric stress tensor and $L^{2}$ is the extensibility parameter. The latter is employed to relate the maximum length of a fully extended dumbbell to its equilibrium length, and here is set to $L^{2}=5000$ to match the molecular weight of the polymer used in the experiments, as explained in Sec. IIE. This viscoelastic model, valid for dilute polymer solutions, exhibits constant shear viscosity and predicts a nonzero, shear-thinning, first normal-stress coefficient which is controlled by the value of $L^{2}$ [72]. Moreover, in steady-state extensional flow, as occurs at the stagnation point in the cross-slot geometry that is examined here, the extensional viscosity predicted by the model exhibits a bounded behavior [72,73].

An in-house implicit, time-marching, finite-volume solver $[74,75]$ is employed in order to numerically solve the governing equations [Eqs. (9)-(12)]. The solver is appropriate for collocated numerical grids, with the convective terms in both the momentum and the polymeric constitutive equation discretized based on the CUBISTA high-resolution scheme [76]. The diffusive terms are discretized considering a central-difference scheme, while the transient terms are evaluated using a first-order implicit Euler scheme. It should be noted that, since we are interested in only the steady-state solution, the first-order accuracy discretization of the transient term is not restrictive, since the time derivative vanishes when steady state is reached.

The bulk of our simulations are performed at constant $\mathrm{Wi}_{\text {eff }}$ (fixed in the range $0.01 \leq \mathrm{Wi}_{\mathrm{eff}} \leq 0.12$ ) on two fluids described by high viscosity ratios $(\beta=0.90$ and $\beta=0.95)$ representative of the experimental samples. By progressive reduction of the Reynolds number from an initially asymmetric solution, a value is obtained for the lower critical Reynolds number $\mathrm{Re}_{c}^{*}$ for which the flow regains symmetry. Since $\mathrm{Wi}_{\text {eff }}$ is fixed while $\mathrm{Re}$ is varied, these simulations involve a varying elasticity number El. In order to more closely mimic some of the experimental (i.e., fixed El) conditions, a few simulations are performed under conditions of constant elasticity number $(\mathrm{El}=0.00018$ with $\beta=0.99$, $\mathrm{El}=0.00083$ with $\beta=0.97, \mathrm{El}=0.0021$ with $\beta=0.95$, and $\mathrm{El}=0.0042$ with $\beta=0.90$ ). In these four cases, ramps are performed both up and down in Re in order to examine whether the hysteresis observed (or not) experimentally can be reproduced by the model. We reiterate for clarity that all of the flow fields solved numerically are steady-state solutions.

\section{RESULTS AND DISCUSSION}

\section{A. Initial experimental observations of inertial and elastic instabilities}

Before proceeding to conduct detailed flow velocimetry experiments, we first perform visualizations of dyeadvection patterns as observed in the $x-y$ center plane of the cross slot at $z=0$. For this we employ a differential
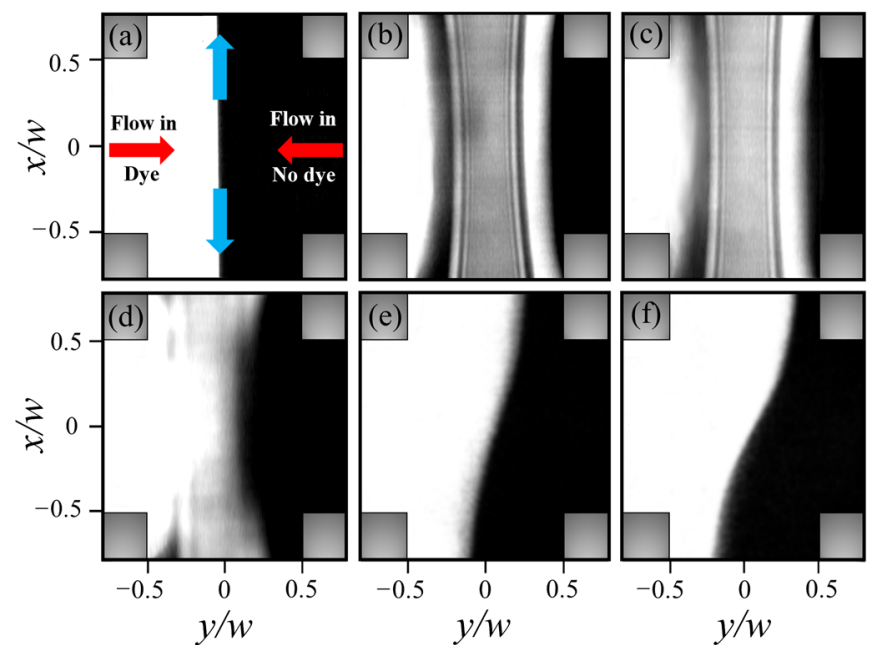

FIG. 3. Confocal microscope images of dye-advection patterns taken in the $x$ - $y$ center plane $(z=0$ plane $)$ of the cross-slot device for aqueous PEO solutions of various El. Fluid dyed with rhodamine B enters from the left-hand inlet channel and undyed fluid enters through the right-hand inlet channel. (a) Stable flow with a symmetric interface (Newtonian or viscoelastic, $\operatorname{Re}<10$ ).

(b) Inertial instability of Newtonian fluid $(\mathrm{El}=0, \mathrm{Re}=80)$.

(c) Inertioelastic instability with 0.001 wt $\%$ PEO solution $(\mathrm{El}=0.00018, \mathrm{Re}=79)$. (d) Inertioelastic instability with 0.01 wt $\%$ PEO solution $(\mathrm{El}=0.011, \mathrm{Re}=33)$. (e) Elastic instability with 0.1 wt $\%$ PEO solution $(\mathrm{El}=0.68, \mathrm{Re}=49)$. (f) Elastic instability with $0.3 \mathrm{wt} \%$ PEO solution $(\mathrm{El}=10.4$, $\mathrm{Re}=15$ ). See also the video corresponding to (f) contained in the Supplemental Material [78].

spinning disk (DSD2) confocal microscope (Andor Technology Ltd, Belfast, UK) and a range of aqueous PEO solutions with various El. A solution containing the fluorescent dye rhodamine $\mathrm{B}(10 \mu \mathrm{M})$ is introduced through one inlet of the channel while an undyed solution is introduced through the other inlet. We confirm that the addition of dye at this concentration has a negligible effect on the physical properties of the fluids.

As shown in Fig. 3(a), at low $\operatorname{Re}(<10)$ a straight and symmetric interface is observed between the dyed and undyed fluid streams entering the cross slot. Similar symmetric flow patterns are observed for all the fluids (Newtonian and polymeric) at this low Re. By contrast, Fig. 3(b) shows what is observed for the Newtonian fluid (water in this case) when a Reynolds number of $R e=80$ is imposed. Here, a distinctive banded structure of alternating bright (dyed) and dark (undyed) regions is observed. This pattern results from the confocal microscope seeing a slice taken along the $z=0$ plane through the spiral vortex that has formed, as was similarly shown by Ait Mouheb et al. [77]. For a PEO solution of $c=0.001 \mathrm{wt} \%$ and $\mathrm{El}=$ 0.00018 at $\operatorname{Re}=79$ [Fig. 3(c)], the dye-advection pattern is similar to that of water at $\mathrm{Re}=80$ [Fig. 3(b)]. As the PEO concentration is increased to $c=0.01 \mathrm{wt} \%(\mathrm{El}=0.011)$, the structure observed at $\operatorname{Re}=33$ [Fig. 3(d)] is significantly 
less clear compared with Figs. 3(b) and 3(c). This is an indication that the flow has become unsteady under these conditions, varying spatiotemporally on a time scale shorter than the image acquisition. At still higher polymer concentrations, $c=0.1 \mathrm{wt} \%[\mathrm{El}=0.68$, Fig. 3(e) $]$ and $c=0.3 \mathrm{wt} \% \quad[\mathrm{El}=10.4$, Fig. 3(f) $]$, the banded pattern indicative of the vortex formation is not observed at all, and instead a different mode of instability emerges. In these images we see that most of the dyed fluid flows out through the upper exit channel, while most of the undyed fluid flows out through the lower exit channel. This flow asymmetry has been reported before and it is considered to be an elasticitydominated flow instability $[29,31,32,35,36]$. We note for completeness that the elastic mode observed in this case is unsteady in time. A video showing this unsteadiness, corresponding to the case shown in Fig. 3(f), is available as Movie M1 in the Supplemental Material [78].

\section{B. Experimental and numerical vorticity fields}

Next, for more quantitative and detailed characterization of the transition to the inertial flow instability, we conduct $\mu$-PIV measurements in the channel cross section at the $y$-z center plane ( $x=0$ plane). In order to validate our technique against previous measurements, we first conduct tests with water for increasing and decreasing Re ramps. The results are shown in Fig. 4. Here, for increasing Re, starting at $\operatorname{Re}=37.1$ [Fig. 4(a)] the flow is steady and symmetric. The two incoming fluid streams from positive and negative $y$ directions meet at $y=0$ and form an essentially straight interface along the $z$ axis. At this Reynolds number, the four cells of Dean vortices previously reported in the numerical simulations of Haward et al. [18] are already apparent in the four quadrants of the channel cross section. In Fig. 4(a), the four Dean vortices are relatively balanced and, hence, the interface is straight and symmetric. As Re is increased [Figs. 4(b) and 4(c)] the flow remains approximately symmetric, with perhaps some tilt of the interface between incoming fluid streams with respect to the $y$ and $z$ axes. This tilt results from an imbalance between the two diagonally opposed pairs of corotating Dean vortices and leads to a small nonzero value of the center point axial vorticity. Finally, for $\operatorname{Re}>\operatorname{Re}_{c} \approx$ 40 a fully developed steady spiral vortex forms, centered on $y=z=0$ and rotating in the anticlockwise direction about the $x$ axis [Fig. 4(d)]. At this stage, the center point axial vorticity jumps greatly in magnitude. As expected for a Newtonian fluid in a cross-slot device of square cross section (i.e., $\alpha=1$ ) [18], hysteresis is observed in the transition. When the Reynolds number is slowly decreased [Figs. 4(e)-4(h)], we find that the flow recovers symmetry only when $\operatorname{Re}<\operatorname{Re}_{c}^{*}=38.8$. In Movie M2 in the Supplemental Material, we present a video depicting the formation and disappearance of the vortex as Re is ramped

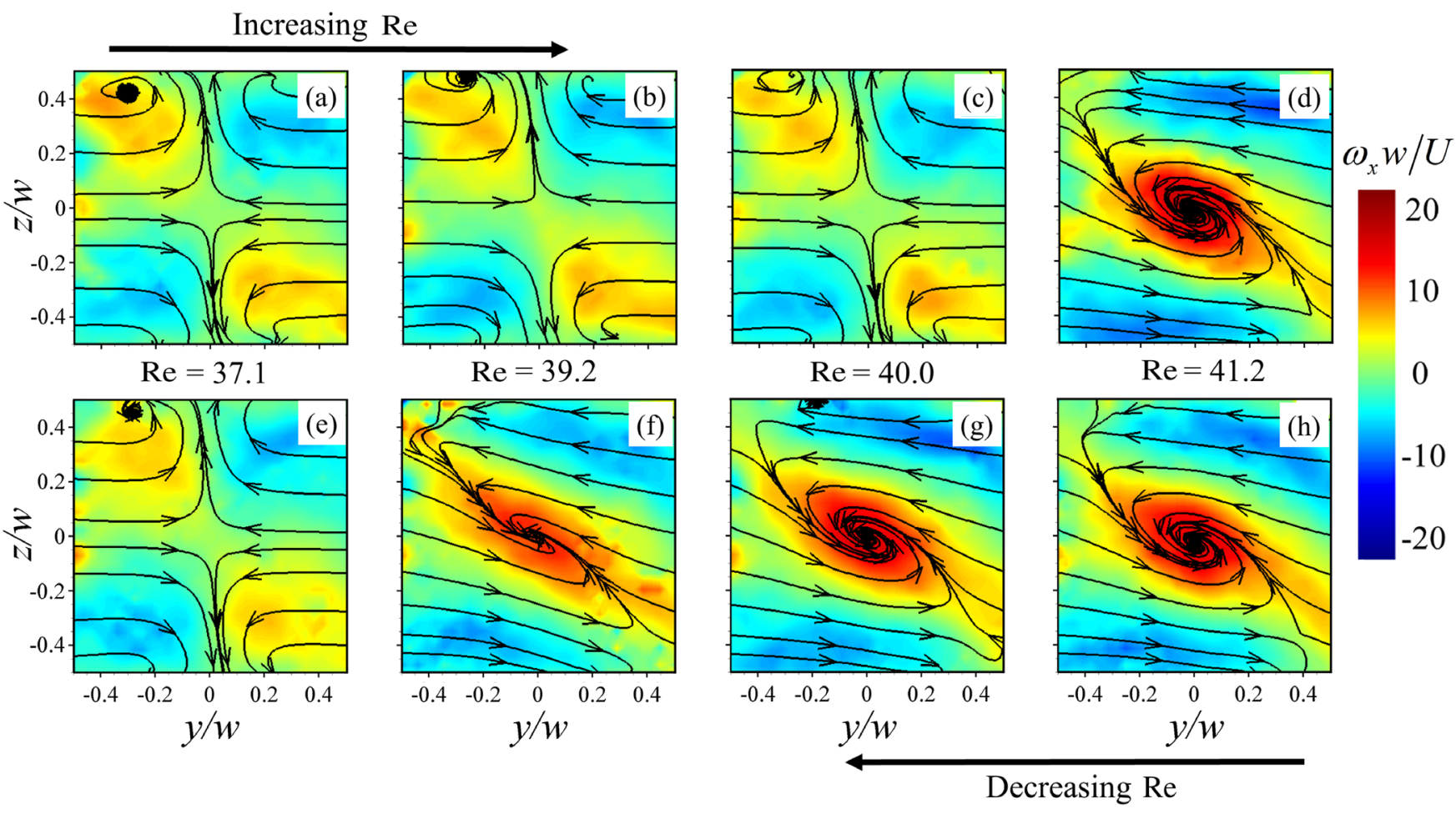

FIG. 4. Experimental $\mu$-PIV images in the $x=0$ plane made using water seeded with fluorescent microparticles. Panels (a)-(d) show results for progressive increases in Re from below the transition, while panels (e)-(h) show the results for progressively decreasing Re from above the transition. (a),(e) $\operatorname{Re}=37.1$; (b),(f) $\operatorname{Re}=39.2$; (c),(g) $\operatorname{Re}=40.0$; (d),(h) $\operatorname{Re}=41.2$. The color scale indicates the local value of the normalized axial vorticity. 

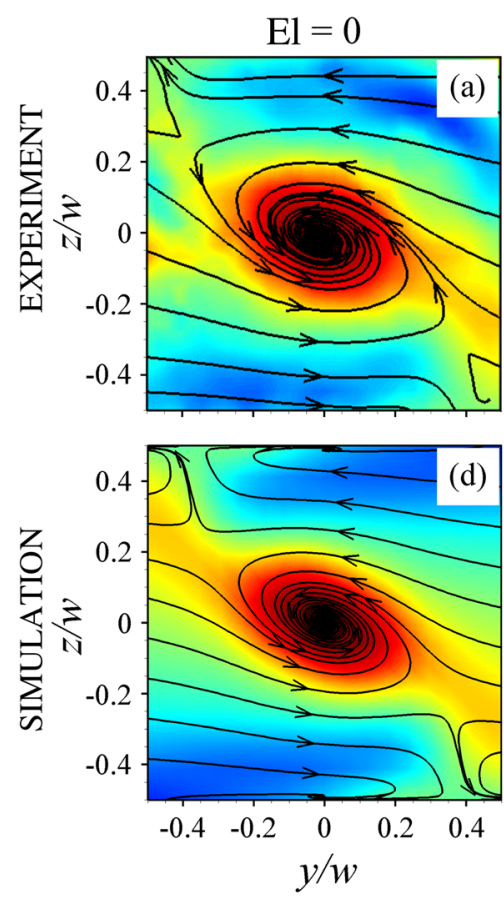
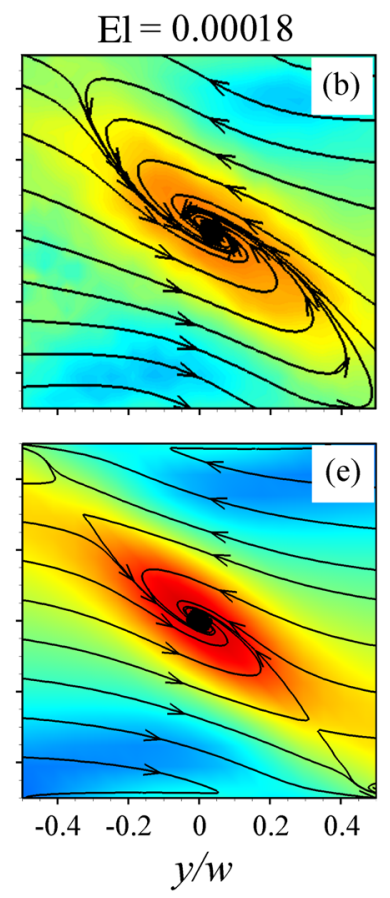
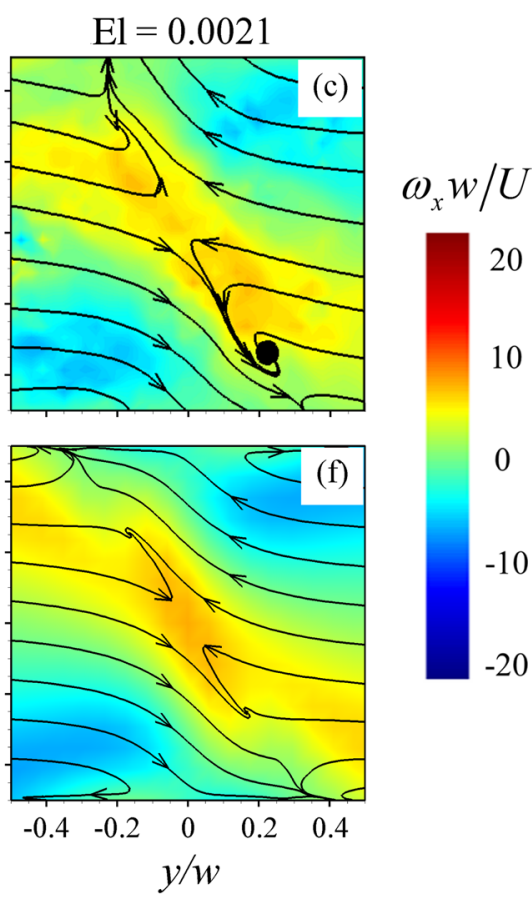

0

0

$-10$

$-20$

FIG. 5. A comparison between experimental measurements and numerical simulations of the dimensionless vorticity $\left(\omega_{x} w / U\right)$ over the $x=0$ plane for fluids of various elasticity number El. Top panels show experimental $\mu$-PIV images obtained at the same value of the dimensionless order parameter $\varepsilon=0.15$ [Eq. (8)]: (a) $c=0 \mathrm{wt} \%$ PEO, Re $=47.2$; (b) $c=0.001 \mathrm{wt} \%$ PEO, Re $=37.0$; (c) $c=0.003 \mathrm{wt} \% \mathrm{PEO}, \mathrm{Re}=28.3$. Bottom panels show converged solutions obtained from constant El numerical simulations with the FENE-MCR model and $L^{2}=5000$ at similar Reynolds numbers to the experiments: (d) $\beta=1$ (Newtonian), Re $=47.0$; (e) $\beta=0.99, \operatorname{Re}=40.0$; (f) $\beta=0.95, \operatorname{Re}=26.2$. The color scale indicates the dimensionless vorticity. Superimposed streamline projections exhibit the directionality of the secondary flow in the cross section.

up and then down over the full range of Re tested [78]. These findings are in close agreement with experimental and numerical observations made on a similar system by Haward et al. [18]. However, we point out that in their work hysteresis in the transition was elucidated from numerical simulations only. In their experiments, which employed laser-scanning confocal microscopy to visualize dyeadvection patterns in the channel cross section, performing quasistatic variations in the Re was not possible and therefore hysteresis could not be resolved [18]. Furthermore, comparisons between the results of the experiments and the numerical simulations were made using different order parameters: a specific local velocity component in the case of the numerical simulations and a dilution-based mixing parameter in the case of the experiments [18]. Here, not only can we resolve the hysteresis experimentally, but we also directly obtain quantitative spatially resolved information on the velocity field, which can be directly compared with our numerical simulations, as we demonstrate in the following.

After confirming that our new experimental setup is valid in comparison with previous experiments with Newtonian fluids, we test several aqueous PEO solutions of different polymer concentrations with different El. Our measurements focus on the Re range that is slightly below and slightly above the flow transition from stable symmetric to asymmetric flow and the formation of a central vortex. Figures 5(a)-5(c) show a series of images for fluids of progressively increasing El taken at a specific value of the dimensionless control parameter, $\varepsilon=\left(\operatorname{Re}-\operatorname{Re}_{c}\right) / \operatorname{Re}_{c}=0.15$. From the images it is strikingly clear that, under comparable flow conditions beyond the onset of instability, the induced vorticity decreases significantly as the fluid elasticity is increased. In Figs. 5(d)-5(f), we present vorticity fields resulting from numerical simulations with the FENE-MCR model under conditions of constant El corresponding to Figs. 5(a)-5(c), respectively. There is a remarkably good, near quantitative, agreement between the experiment and the numerical simulations.

In Fig. 6, we present experimental vorticity fields obtained at $\varepsilon=0.15$ for two additional aqueous PEO solutions of higher polymer concentration and elasticity $(c=0.01 \mathrm{wt} \%$, $\mathrm{El}=0.011$, and $c=0.03 \mathrm{wt} \%, \mathrm{El}=0.078)$. Under these conditions there is unsteadiness in the flow; the position of the vortex and the magnitude of the vorticity fluctuates slightly in time. Since numerical simulations are restricted to steady-state solutions (see Sec. II H), comparable numerical results at these elasticity numbers are not available. The images shown in Fig. 6 are in fact averages made of 15 individual vector fields captured at a rate of $5 \mathrm{~Hz}$ over a 3-s time interval. In Movies M3 and M4 in the Supplemental Material, we present the respective sequences of 15 images in video format [78]. Note that the spatiotemporal fluctuations 


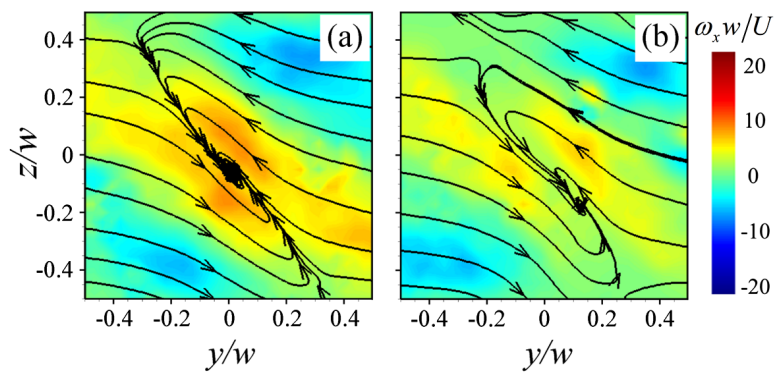

FIG. 6. Experimental dimensionless vorticity fields in the $x=0$ plane at $\varepsilon=0.15$ for (a) $c=0.01 \mathrm{wt} \% \mathrm{PEO}$ in water $(\mathrm{El}=0.011, \mathrm{Re}=19.7)$ and $(\mathrm{b}) c=0.03 \mathrm{wt} \%$ PEO in water $(\mathrm{El}=0.078, \mathrm{Re}=17.2)$. Under these conditions the flow exhibited mild unsteadiness and the images shown are averaged over 15 individual fields. The color scale indicates the dimensionless vorticity $\left(\omega_{x} w / U\right)$. Superimposed streamlines exhibit the directionality of the flow. See also the videos contained in the Supplemental Material [78].

we report in these two fluids with $\mathrm{El} \ll 1$ are completely absent in a Newtonian fluid at an equivalent value of $\varepsilon=0.15$, for which both experiments and simulations clearly show the flow remains steady and laminar.

In Fig. 7, we present the order parameter $\psi$ [Eq. (7)] as a function of the applied Reynolds number for a range of aqueous PEO solutions with elasticity numbers $0 \leq \mathrm{El} \leq 0.68$. Here, closed symbols represent data obtained by applying quasistatic increases in Re, while open symbols represent data obtained from a subsequent quasistatic decreasing ramp in Re. For each fluid, as Re is progressively increased starting from a low value, $\psi$ initially adopts a value close to zero, indicating that the flow field is symmetric. As a fluid-dependent critical value $\operatorname{Re}_{c}$ is reached, the flow field adopts an asymmetric state, resulting in the measurement of a nonzero axial vorticity $\omega_{x}$ at the center point $(y=z=0)$ and, hence, a nonzero value of the order parameter $\psi$. For subsequent further increases in $\operatorname{Re}$ above $\operatorname{Re}_{c}$, the measured center point vorticity continues to increase towards a plateau value as the central spiral vortex develops. For subsequent quasistatic reductions in $\operatorname{Re}$ starting from a high value (i.e., $\operatorname{Re}>\operatorname{Re}_{c}$ ), the center point vorticity also reduces, following the same curve defined by the data for quasistatic increases in Re. For some of the experimental cases (though not all), there is hysteresis in the transition and Re must be reduced to a value $\operatorname{Re}_{c}^{*}<\operatorname{Re}_{c}$ before the flow field recovers symmetry. The occurrence (or otherwise) of hysteresis appears to have a complex nonmonotonic dependence on the concentration of PEO in the polymer solution, or on the elasticity number of the experiment. We return to this point in more detail in a subsequent section of the discussion. We note that in the numerical simulations hysteresis is always observed in the transition, and that the value of $\operatorname{Re}_{c}$ tends to be slightly higher than what is observed in the experiments. We find that the flow transition from symmetric to an asymmetric

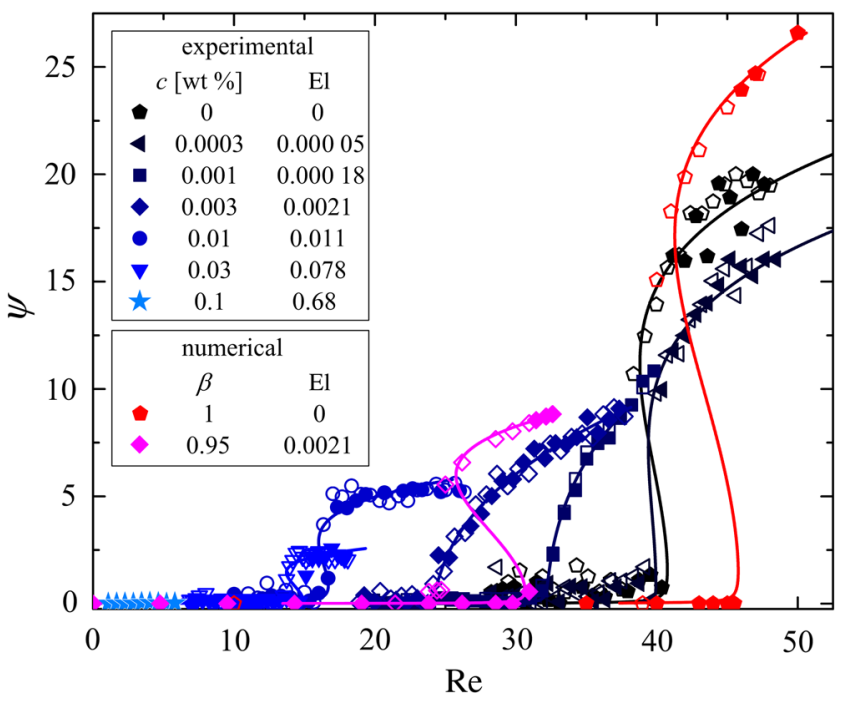

FIG. 7. Order parameter $\psi$ as a function of Re for solutions of PEO in water, with the viscosity ratio spanning $0.24<\beta<1$ for the experimental fluids. Closed symbols indicate data obtained with increasing flow rates and open symbols indicate data obtained with decreasing flow rates. Data are fitted with the Landau model [Eq. (8), solid lines]. Numerical data are obtained with the FENE-MCR model with $L^{2}=5000$ and constant El.

state in the simulations for increasing Re depends on the level of numerical noise (e.g., mesh size, times step). However, for decreasing Re ramps, the value obtained for $\mathrm{Re}_{c}^{*}$ is more reliable numerically and more consistent with the experiments, as was also found in our previous work [18]. For this reason, further comparison between the experimental and the numerical results will be made on the basis of decreasing Re ramps. Despite the aforementioned minor differences, there is clearly a good general consistency between the experimental and the numerical data presented in Fig. 7. It is immediately obvious from both data sets that, as El is increased, the value of $\operatorname{Re}_{c}$ is decreased. In addition, the normalized center point vorticity tends to approach a reduced plateau value with increasing El. In fact, in our experiments with a $c=0.1 \mathrm{wt} \%$ PEO solution $(\mathrm{El}=0.68)$ we do not observe any increase in the center point vorticity before the onset of the elasticitydominated flow asymmetry [as illustrated by Fig. 3(e)]. Curve fitting of the data in Fig. 7 at each fixed value of $\mathrm{El}$ is performed using the Landau model [Eq. (8)].

Additional $\mu$-PIV measurements are performed using solutions of PEO in the more viscous solvent composed of $8 \mathrm{wt} \%$ aqueous PEG. This allows us to formulate fluids with relatively long relaxation times while avoiding the effects of shear thinning, which occur in the purely aqueous PEO solutions at higher concentrations. Thus, we are able to clearly isolate the importance of fluid elasticity from shear thinning in the polymer solutions. Figure 8(a) shows a sequence of four images taken over a range of $\mathrm{Re}$ spanning the onset of the transition for the flow of a $c=$ $0.0001 \mathrm{wt} \%$ solution of PEO in the $8 \mathrm{wt} \%$ PEG Newtonian 

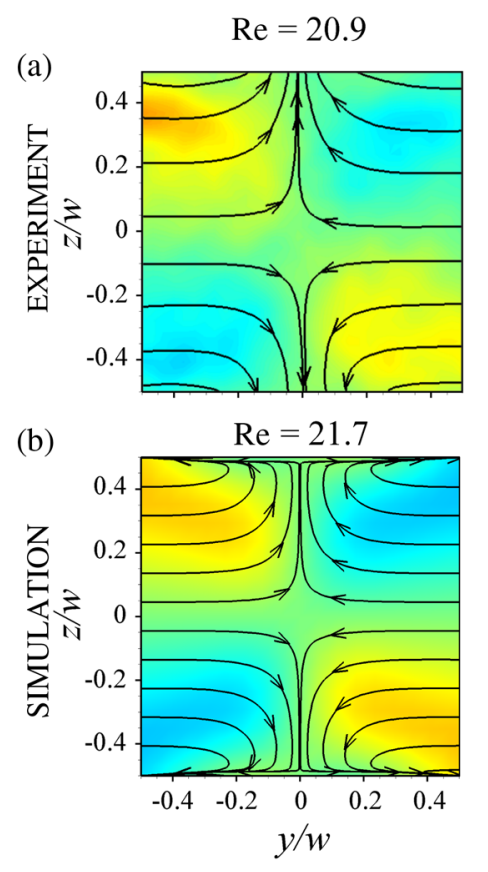
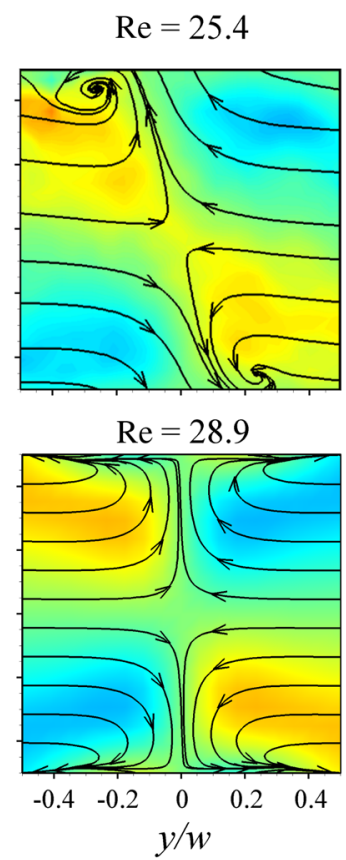

$\operatorname{Re}=31.4$
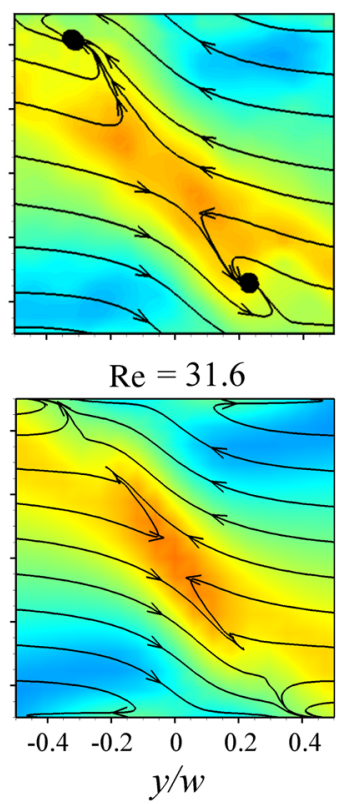

$\operatorname{Re}=38.6$
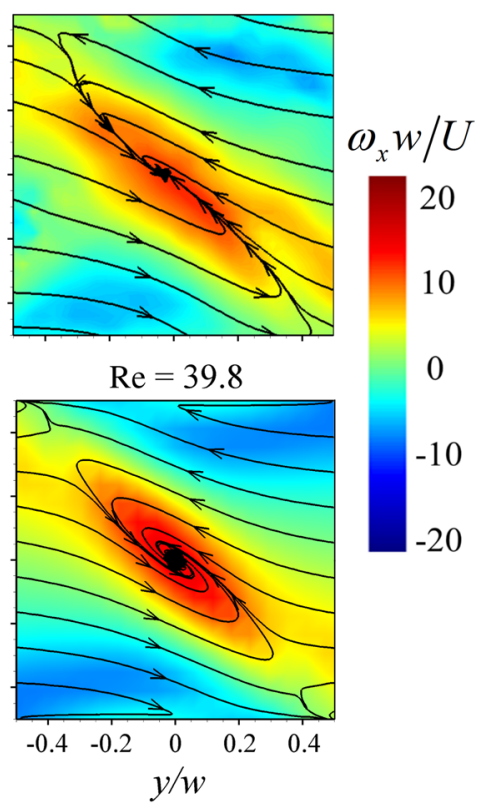

FIG. 8. A comparison between (a) experimental measurements with a $c=0.0001 \mathrm{wt} \%$ solution of PEO in 8 wt $\%$ PEG $(\beta=0.97$, $\mathrm{El}=0.00083$ ) and (b) numerical simulations with $L^{2}=5000$ and $\beta=0.97$ at constant $\mathrm{El}=0.00083$. The color scale indicates the dimensionless vorticity $\omega_{x} w / U$ in the $x=0$ plane. Projected streamlines show the directionality of the secondary flow in the cross section.

solvent. Here, we only show data obtained for quasistatic decreases in Re. This sequence can be compared with Figs. 4(e)-4(h) for the flow of a Newtonian fluid, revealing marked differences even at this low polymer concentration (only 1 part per million). The flow field in the PEO solution remains asymmetric down to a significantly lower Reynolds number than for the Newtonian fluid, and the vorticity intensification at higher $\mathrm{Re}$ is noticeably perturbed in the weakly elastic fluid. Figure 8(b) shows a sequence of images obtained from a numerical simulation designed to mimic the experiment shown in Fig. 8(a) and, once again, results in generally good qualitative agreement.

Differences between the low concentration PEO solutions and the $8 \mathrm{wt} \%$ PEG Newtonian solvent are clearer to see in Fig. 9, which shows the order parameter $\psi$ measured for increasing and decreasing $\mathrm{Re}$ and fitted using the Landau model given in Eq. (8). The $8 \mathrm{wt} \%$ PEG solvent behaves quite similarly to the pure water (Fig. 7), showing subcritical behavior with a characteristic hysteresis in the bifurcation. The critical Reynolds number for quasistatic increases in $\operatorname{Re}$ is $\operatorname{Re}_{c}=40.0$, while for decreasing $\operatorname{Re}$ the transition occurs for $\operatorname{Re}_{c}^{*}=38$.0. Further, for $\operatorname{Re}>\operatorname{Re}_{c}$ the dimensionless vorticity for both water and $8 \mathrm{wt} \%$ aqueous PEG approach similar values. The encouraging self-similar behavior displayed by the two Newtonian fluids gives good confidence that we are correctly nondimensionalizing our order and control parameters. Also (similarly to the experiments performed using PEO solutions prepared in pure water), as the elasticity number increases, the bifurcation occurs at a progressively lower value of Re and a general reduction in the maximum center point vorticity occurs. At the highest concentration of PEO in the aqueous $8 \mathrm{wt} \%$ PEG solvent that we measure $(0.003$ wt $\%, \mathrm{El}=0.34)$, we observe no clear increase in the value of $\psi$ over the range of

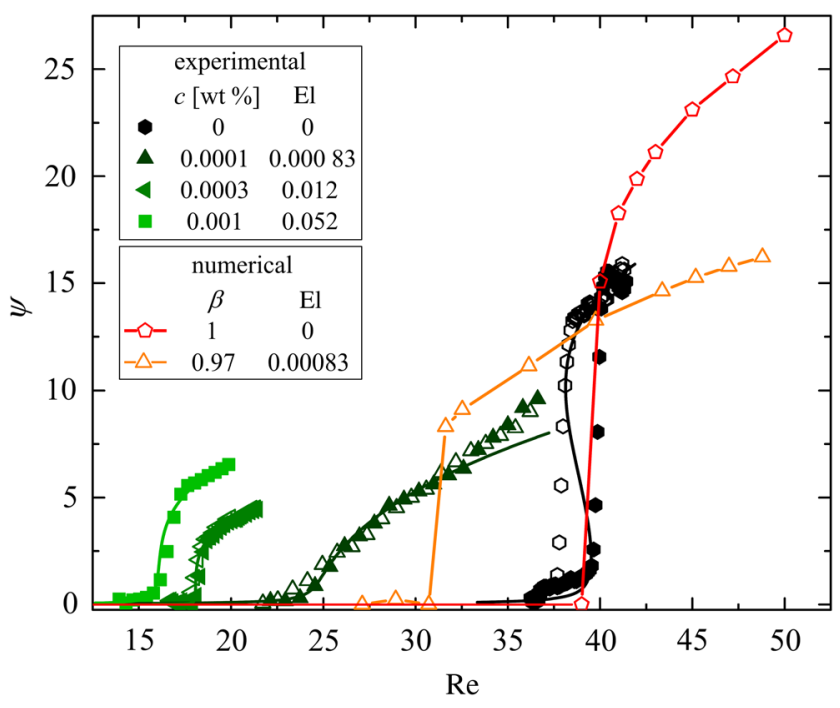

FIG. 9. Order parameter $\psi$ as a function of Re for solutions of PEO in 8 wt \% PEG, with the viscosity ratio spanning $0.91<$ $\beta<1$ for the experimental fluids. Closed symbols indicate data obtained with increasing flow rates and open symbols indicate data obtained with decreasing flow rates. Data are fitted with the Landau model [Eq. (8), solid lines]. Numerical data (decreasing Re ramp only) are obtained with the FENE-MCR model with $L^{2}=5000$ and constant El. 
Re we test. Instead, we observe the elasticity-dominated mode of instability characterized by the asymmetric flow state, as exemplified by Figs. 3(e) and 3(f) (data not shown).

\section{Detailed analysis and phase diagrams}

In this section, we summarize the results obtained from both our experiments and our numerical simulations in the form of phase diagrams in dimensionless parameter space. In Fig. 10, we present the values of the lower critical Reynolds numbers $\mathrm{Re}_{c}^{*}$ for the inertial flow transition as a function of the elasticity number El. Over the full range of $\mathrm{El}$ for which inertia-dominated transitions occur, the values of $\mathrm{Re}_{c}^{*}$ obtained from both experimental fluid systems (water and $8 \mathrm{wt} \%$ PEG solvents) collapse well onto a single curve described by a strongly decaying exponential function of $\mathrm{El}: \operatorname{Re}_{c}^{*}=25 \exp (-250 \mathrm{El})+15$. Furthermore, at the low values of $\mathrm{El}<0.01$, which give rise to a steady flow bifurcation, the critical Reynolds numbers obtained from the numerical simulations also collapse onto the same curve.

The exponential decay of $\operatorname{Re}_{c}^{*}$ with increasing $\mathrm{El}$ indicates a system in which the sensitivity to elasticity increases exponentially as $\mathrm{El}$ is reduced. This suggests the potential utility of the system as a novel rheometer for weakly elastic fluids. The concept has some analogy with the microfluidic serpentine channel rheometer of Zilz et al., [79], but here exploiting an inertia-dominated as opposed to a purely elastic instability.

We remark on the similarity between the form of Fig. 10 and the reduction in critical Reynolds number with increasing polymer concentration reported for the onset of EIT by

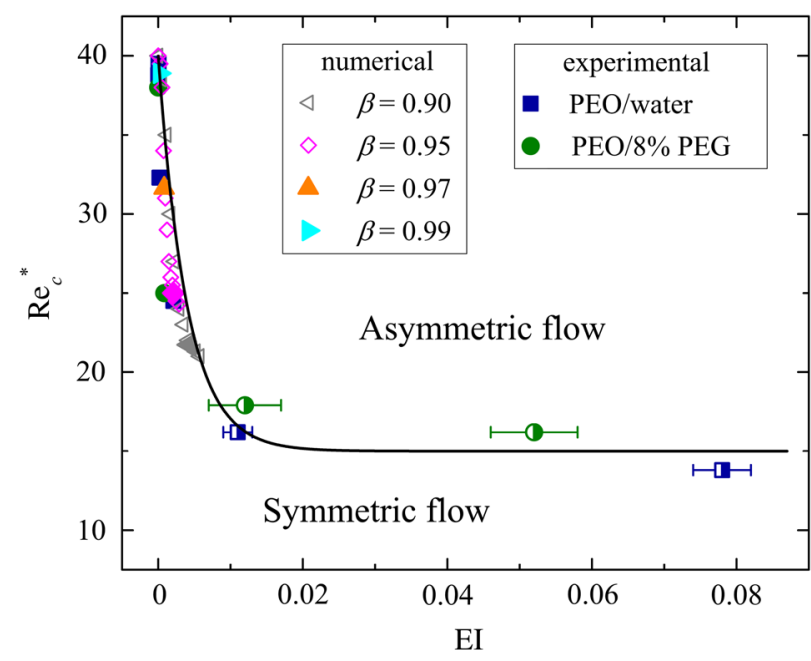

FIG. 10. Lower critical Reynolds number $\operatorname{Re}_{c}^{*}$ as a function of the elasticity number El for inertia-dominated flow instabilities of low concentration PEO solutions in a cross-slot device with $\alpha=1$. For experimental data, closed symbols represent steady instabilities, while half-closed symbols represent unsteady instabilities. For numerical results, open symbols represent constant $\mathrm{Wi}_{\text {eff }}$, while closed symbols represent constant El simulations.
Samanta et al. [4], although the onset Reynolds number for vortex formation in the cross slot is around 2 orders of magnitude smaller than that for EIT.

The critical Reynolds number for the onset of inertioelastic flow instabilities in T-shaped intersecting channels with two inlets of aspect ratio $\alpha=1$ and one outlet of $\alpha=2$ has also been found to be dependent on El [80]. In the Newtonian case, flow in such channels becomes unstable, resulting in the formation of vortices extending along the outlet channel when the Reynolds number exceeds a critical value $\operatorname{Re}_{c} \approx 100[53,54,81]$. There are rather few numerical or experimental studies of non-Newtonian flows in $\mathrm{T}$ channels [80,82-84]. However, using the upperconvected Maxwell model, Poole et al. showed numerically that low levels of fluid elasticity could cause a reduction in $\operatorname{Re}_{c}$ [80]. With highly elastic fluids $(\mathrm{El}=861)$, Soulages et al. [83] reported flow asymmetries in microfluidic $\mathrm{T}$ channels that appear closely related to the purely elastic asymmetries seen in cross-slot devices [31,32]. These limited reports suggest that the effects of increasing fluid elasticity may be similar in various different types of intersecting geometries containing stagnation points.

As we mention in Sec. III B in the discussion of Figs. 7 and 9, the Newtonian solvents and some of the polymeric test fluids show hysteretic behavior with a critical Reynolds number that depends on whether the flow rate is ramped up or down quasistatically. Figure 11 summarizes the appearance of hysteresis in the transition for all the experimental test fluids as a function of the elasticity number. Here, we examine the ratio of the parameters $g$ and $k$ obtained by fitting the Landau model to the experimental data. The ratio $g / k$ has a negative value for Newtonian fluids $(\mathrm{El}=0)$, indicating a subcritical transition with hysteresis. As El increases, the size of the hysteresis loop decreases and the

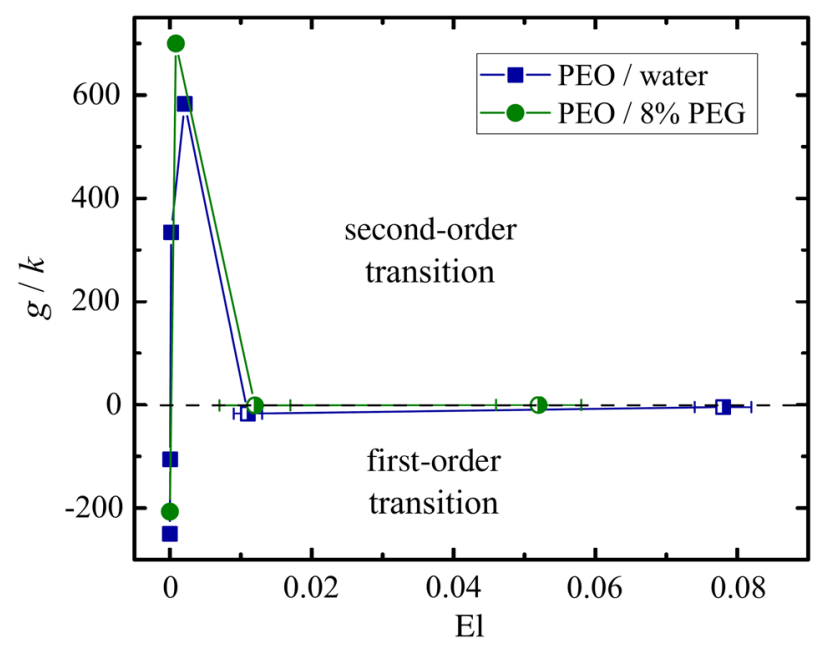

FIG. 11. The ratio of the parameters $g$ and $k$ as a function of El. The dashed line indicates $g=0$, where the transition would be tricritical. Above the line the transition is supercritical; below the line the transition is subcritical with hysteresis. 
transition turns apparently supercritical. For a specific intermediate value of El where $g / k=0$, the transition would be tricritical. The transition remains supercritical up to values of $\mathrm{El} \approx 0.01$. For fluids with elasticity values between $0.01<\mathrm{El}<0.09$, certain fluids have slightly negative values of $g / k$ and show small hysteresis loops (see, e.g., Fig. 7), but the general tendency is towards tricritical behavior. Of course, the sensitivity of the experiment to noise at different El levels may be masking any underlying hysteretic behavior. In contrast, the numerical simulations with the FENE-MCR model demonstrate hysteretic transitions for all elasticity numbers and $\beta$ values tested.

Changes in the order of the transition through a tricritical point have been reported in previous work with Newtonian fluids in the cross-slot geometry when the parameter space was changed geometrically by changing the aspect ratio $\alpha$ [18]. Tricritical phenomena in Newtonian fluid mechanics have also been reported for flow in low aspect ratio TaylorCouette flow cells [85-87].

Studying flow transitions in non-Newtonian fluids is more complex compared with Newtonian fluids due to the additional dimensions of the parameter space [88]. NonNewtonian transitions in the cross slot depend not only on $\mathrm{Re}$ and $\alpha$ but also on Wi and $\beta$ (and potentially other groups, such as the ratio between first and second normal stress coefficients). This additional parameter space is accessed and explored by changing the $\mathrm{El}$ of the fluid. Here, this is achieved through manipulation of the polymer concentration and the solvent viscosity, but similar variations could be made via control of, e.g., the polymer molecular weight, polymer flexibility, or solvent quality.

In this work, elastic effects in the fluids are characterized using the effective Weissenberg number $\mathrm{Wi}_{\text {eff }}$, Eq. (5) (which factors out both $\lambda$ and $\beta$ ), and the elasticity number El, Eq. (6) (which additionally factors out Re, i.e., length scale, viscosity, and density). In Fig. 12, we show the values of the lower critical effective Weissenberg number $\mathrm{Wi}_{\mathrm{eff}, c}^{*}$ as a function of $\mathrm{El}$ for all the polymeric test fluids listed in Table II. For fluids that display inertia-dominated (or inertioelastic) instabilites, the lower critical effective Weissenberg number is computed as $\mathrm{Wi}_{\mathrm{eff}, c}^{*}=\mathrm{Re}_{c}^{*} \times \mathrm{El}$. For the fluids that show elasticity-dominated flow asymmetries, $\mathrm{Wi}_{\mathrm{eff}, c}^{*}$ is estimated from the results of the coarse dye-advection experiments illustrated in Fig. 3. All of the data collapse onto a power law with exponent 0.8 , as shown by the straight line through the experimental and numerical data points on the log-log plot. We observe clearly that the elastic mode of instability dominates as the elasticity number approaches the value $\mathrm{El}=1$, as might be expected [89].

Increasing $\mathrm{El}$ influences the inertial instability by increasing the $\mathrm{Wi}_{\text {eff }, c}$ at which the transition occurs. Higher El further reduces the relative importance of inertial forces, and as El approaches the value 1, the inertial instability is completely suppressed, giving rise instead

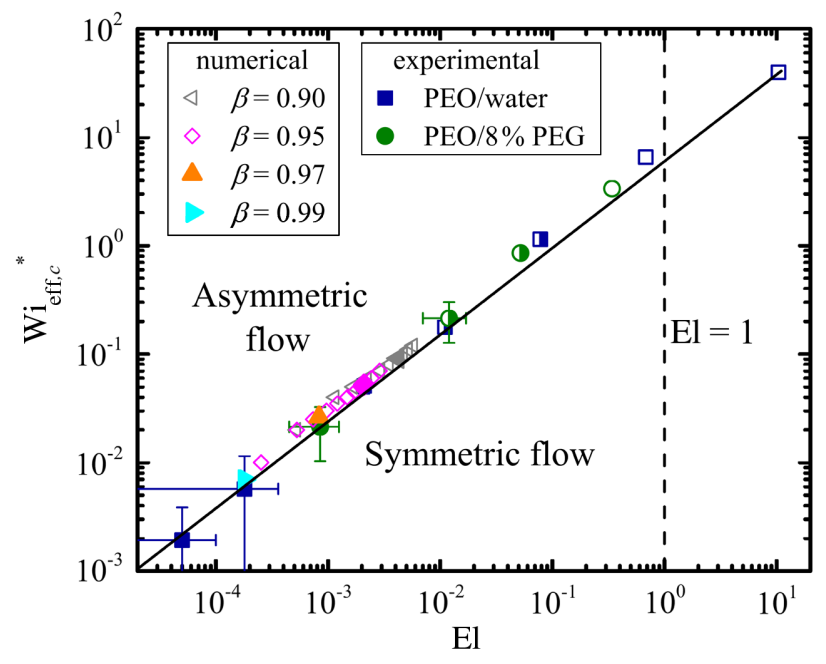

FIG. 12. $\quad \mathrm{Wi}_{\text {eff,c }}^{*}$ as a function of El. For experiments, closed symbols represent steady spiral instabilities, half-closed symbols represent unsteady spiral vortex instabilities, and open symbols represent elasticity-dominated asymmetries. For numerical results, open symbols represent constant $\mathrm{Wi}_{\text {eff }}$, while closed symbols represent constant $\mathrm{El}$ simulations. The dashed line marks $\mathrm{El}=1$. Power-law fit through the data is $\mathrm{Wi}_{\mathrm{eff}, c}^{*}=6 \mathrm{El}^{0.8}$. (Note that error bars on the two lowest El experimental data points extend beyond the boundaries of the plot.)

to the purely elastic time-dependent flow instability, as demonstrated in Figs. 3(e) and 3(f).

In Fig. 13, we plot the critical conditions determined for all the non-Newtonian test fluids in the form of a stability diagram in $\mathrm{Wi}_{\text {eff }}-\mathrm{Re}$ dimensionless state space. Here, we represent three flow regimes: (1) a region of stable symmetric flow at lower values of $\mathrm{Wi}_{\text {eff }}$ and Re; (2) a region of inertially dominated spiral-vortex-type instabilities, which dominate at higher Re but for $\mathrm{El} \lesssim 1$; (3) a region at lower $\mathrm{Re}$ and higher $\mathrm{Wi}_{\mathrm{eff}}$, where elastic asymmetries dominate at $\mathrm{El} \gtrsim 1$. The results of numerical simulations deviate somewhat from the experiments at high $\mathrm{Re}$ and low $\mathrm{Wi}_{\text {eff }}$ (i.e., low $\mathrm{El}$ ), but interestingly approach the experimental trend for lower Re and higher $\mathrm{Wi}_{\text {eff }}$ (i.e., as $\mathrm{El}$ increases). We note some similarity of the stability diagram presented in Fig. 13 with those presented by Joo and Shaqfeh [90], who demonstrated by linear stability analysis on Dean and Taylor-Couette flows the destabilization of inertial instability modes by increasing the elasticity, and conversely the destabilization of elastic instability modes by the increase of inertia. We point out that the ranges of $\mathrm{Re} \lesssim 50$ and $\mathrm{Wi}_{\text {eff }} \lesssim 50$ covered in Fig. 7 can be routinely achieved with low viscosity aqueous viscoelastic fluids, even in microfluidic channels. For the validity of such experiments (e.g., cell sorting, immunoassays, DNA analysis), it is clearly of great importance to be aware of the likelyhood of flow instabilty onset beyond critical conditions and to limit the dimensionless flow parameters within bounds where the flow field remains stable and well defined. 


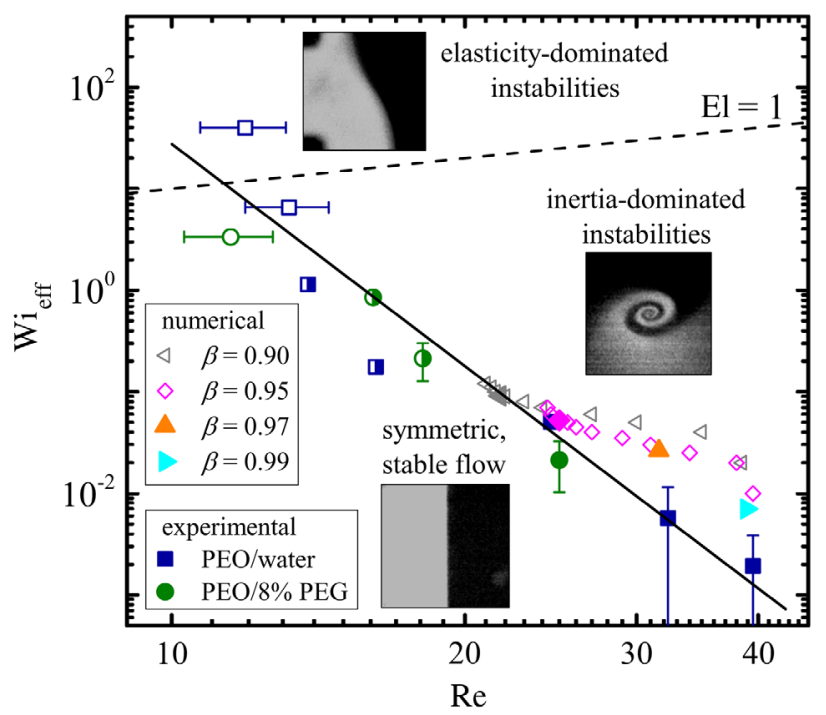

FIG. 13. Stability diagram in dimensionless $\mathrm{Wi}_{\text {eff }}-\mathrm{Re}$ parameter space. A stable region is indicated below the solid line, drawn through the experimental data as a guide to the eye. For experiments, closed symbols represent steady spiral vortex instabilities, half-closed symbols represent unsteady spiral vortex instabilities, and open symbols represent elasticity-dominated asymmetries. For numerical results, open symbols represent constant $\mathrm{Wi}_{\text {eff }}$, while closed symbols represent constant El simulations.

In the cross slot, it has been shown that the instability of Newtonian flows is a consequence of the introduction of a center point axial vorticity due to imbalances between four cells of Dean vortices that form due to centrifugal forces around the corners of the cross slot as inertia becomes significant [18]. For $\operatorname{Re}>\operatorname{Re}_{c, \text { Newt }} \approx 40(\alpha=1)$, vortex stretching drives the growth of the center point vorticity until a steady state is reached. For the weakly elastic fluids examined here, our numerical simulations give insight into how the presence of the polymeric additive modifies the Newtonian instability mechanism by showing the regions of the flow field where the localized orientation of polymer gives rise to increases in the first normal stress difference, $N_{1}=\left(\tau_{x x}-\tau_{y y}\right)$. Such an in situ measurement of $N_{1}$ is not possible using currently available experimental techniques, so the use of complementary simulations here provides a great advantage. Figure 14 shows contours of $N_{1}$ for the case $\mathrm{El}=0.0042, \beta=0.90$. At relatively low Re [Fig. 14(a), $\mathrm{Wi}_{\text {eff }}=0.02, \mathrm{Re}=4.76$ ), the first normal stress difference exhibits the anticipated behavior of an extension-dominated flow, where a thin strand of high stress is formed along the flow centerline emanating from the stagnation point $[32,35,49,91]$. This indicates that significant stretching and orientation occurs at the region close to the stagnation point, which has been demonstrated in previous experiments by the observation of "birefringent strands" [89]. As $\mathrm{Wi}_{\text {eff }}$ and, consequently, Re are increased, the flow field is modified and the highest values of the first normal stress difference are no longer located near the stagnation point, but rather are shifted along the vertical direction $(z)$ forming two peaks close to the top and bottom walls of the channel, as shown in Fig. 14(b). Although in both Figs. 14(a) and 14(b) the flow field remains symmetric, the difference is that when $\mathrm{Wi}_{\mathrm{eff}}=0.08$ there are Dean vortices present due to inertia. Examining the superimposed streamlines in Fig. 14(a), it can be seen that for $\mathrm{Wi}_{\text {eff }}=0.02$ the fluid elements that pass close to the stagnation point, which consequently exhibit the higher stretching, are then almost immediately oriented along the outlet streamwise direction. Thus, as they flow far from the high stretching region they gradually relax and the intensity of the birefringent strand slowly decays. In contrast, for $\mathrm{Wi}_{\text {eff }}=0.08$ the fluid elements along the inlet flow centerline that pass close to the stagnation point are initially stretched and are then oriented to flow towards the $z$ direction of the configuration. When flowing along this path, the already stretched fluid elements experience some additional stretching from the incoming streams, which results in an accumulation of stress from the stagnation point region towards the $z$ direction and generates high-stress
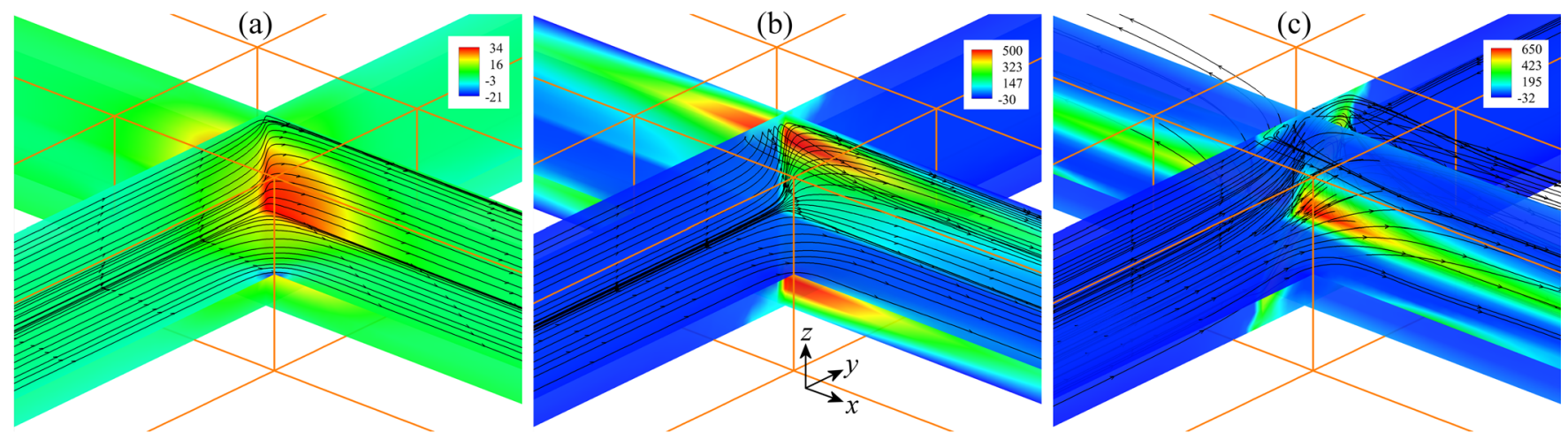

FIG. 14. Contours of the normalized first normal stress difference $N_{1} /\left(\eta_{0} U / w\right)$ (indicated by the color scale) with superimposed streamlines along the center planes of the cross slot. Constant elasticity number simulations using the FENE-MCR model with $\mathrm{El}=0.0042, \beta=0.90$ and (a) $\mathrm{Wi}_{\text {eff }}=0.02, \mathrm{Re}=4.76$ (symmetric), (b) $\mathrm{Wi}_{\text {eff }}=0.08, \mathrm{Re}=19.05$ (symmetric), and (c) $\mathrm{Wi}_{\text {eff }}=0.125$, $\operatorname{Re}=29.76$ (asymmetric). 
peaks located precisely between the pairs of counterrotating Dean vortices. Flow feedback in the region of the Dean vortices by these localized peaks in $N_{1}$ is the likely cause of the destabilization of the flow for $\operatorname{Re}_{c}<\operatorname{Re}_{c \text {,Newt }}$, as observed both experimentally and numerically. Finally, once the central spiral vortex has formed, the region of the highest $N_{1}$ relocates to the vortex core [see Fig. 14(c)]. It is already well known that the polymer torque resulting in such a situation acts counter to the vorticity [92-96], which explains why the vorticity growth is suppressed in the weakly elastic fluids compared with the Newtonian case as Re is increased above $\operatorname{Re}_{c}$. Such a mechanism is thought to be responsible for suppression of streamwise and hairpin vortices in polymer drag-reduced flows $[92,93]$. These numerical results reveal a complex interplay between inertia and elasticity, suggesting that El alone is insufficient to fully capture the observed phenomena, and highlighting that both Wi and Re or (Wi and El) remain important.

For pipe flows at high $\operatorname{Re}(>1000)$, Samanta et al. showed that the criticial Re for the onset of EIT decreased as the polymer concentration was increased [4]. Using pipes of various diameters, they also showed collapse of the critical deformation rate for the onset EIT at different polymer concentrations. The latter observation suggests the importance of polymer deformation and elastic stresses on driving the onset of instability. As Re was increased beyond the onset of EIT, Samanta et al. showed that the friction factor directly approached the maximum drag reduction asymptote while streamwise vortices characteristic of Newtonian turbulence were suppressed. Here, in a very different flow configuration, we make highly analogous observations in a single streamwise vortex as $\mathrm{Re}$ is increased beyond the onset of inertioelastic instability: i.e., a critical $\mathrm{Re}$ that decreases with increasing El, a critical Wi that scales with El, and a suppression of vorticity growth as the flow is driven beyond the onset of instability. Combined with simulations that provide details of the elastic stresses within the vortex, we can clearly rationalize the drag reduction reported by Samanta et al. in terms of the action of the polymer. By using a range of fluids with wellcharacterized elasticity numbers, the collapsed data we obtain for the critical values of $\mathrm{Re}$ and $\mathrm{Wi}_{\text {eff }}$ show that these effects have significant generality. In the cross-slot device, following the onset of inertioelastic instability, the resulting flow structures for very low elasticity fluids appear qualitatively similar to those obtained in unstable Newtonian flows [see, e.g., Figs. 3(b), 3(c), 5(a), and 5(b)]. In this case, the effect of the polymer may be interpreted as causing modification to the inertial (Newtonian) flow state. However, as the elasticity is increased, spatiotemporal fluctuations of the flow structures (which are absent in Newtonian fluids) are observed [see Figs. 3(d) and 6 and Supplemental Material [78] ], and as $\mathrm{El} \rightarrow 1$, we see the emergence of a distinct elasticity-dominated flow state [see Figs. 3(e) and 3(f) and Supplemental Material [78] ]. The fluctuating inertioelastic state shown in Figs. 3(d) and 6 may well be connected to the distinct nature of the fluctuations observed in EIT compared with traditional Newtonian turbulence, reported by Samanta et al. [4]. However, further time-resolved investigations of fluctuations arising at higher Re in Newtonian and inertioelastic flows in the cross-slot geometry will be required in order to confirm this likelihood.

\section{SUMMARY}

In this work, we use state-of-the-art microfabrication methods to reconfigure the canonical cross-slot geometry, enabling the first quantitative measurements of velocity components over a channel cross section, and thus to resolve the axial vorticity in the outlet. This facilitates a unique study on the interactions between an inertial flow instability (that results in the formation of a streamwise vortex along the outlet channel) and the elasticity introduced through the addition of small quantities of a high molecular weight polymer at concentrations relevant to polymer drag reduction. For small increasing values of the elasticity number El, the flow is destabilized at a lower critical Reynolds number than in the Newtonian case. However, following the onset of instability, the growth of the axial vorticity with increasing Re is significantly suppressed by the increase of El. In this regime of low El, our experimental data are well matched by numerical simulations using the constant viscosity FENE-MCR model, which further allows us to locate the regions in the flow domain where high polymer stretch and stress occur. Feedback between localized regions of high polymeric stresses and the flow field are deemed responsible for the destabilization of the flow at a lower critical Reynolds number than seen in the Newtonian case. Suppression of the subsequent vortex formation is most likely through the action of polymer torque. These quantitative measurements at the scale of a single vortex provide clear mechanisms for (and a clear visual demonstration of) how polymer additives potentially act to reduce drag in turbulent flows. As the elasticity is increased towards $\mathrm{El} \sim 1$, our experimental results show how an inertia-dominated instability is transformed into an elasticity-dominated mode as the vorticity becomes completely suppressed by the action of the polymer additive. Our experimental measurement methods can be readily extended to study the dynamics of vortex formation and interactions, and also to study similar 3D and time-dependent flow phenomena in, for example, T- or Y-shaped junctions [53,54,56,57] or L- and U-shaped bends (e.g., Ref. [97]). Our data significantly add to the literature on the stability of inertioelastic flows, which are relevant to understanding a number of important practical applications (e.g., jet breakup, drag reduction, enhanced oil recovery). Our results and techniques are anticipated to provide insight into the stability of weakly elastic fluid flows through intersections and near stagnation points in general. 


\section{ACKNOWLEDGMENTS}

N. B., A. Q. S., and S. J.H. gratefully acknowledge the support of Okinawa Institute of Science and Technology Graduate University, with subsidy funding from the Cabinet Office, Government of Japan. A. Q. S. and N. B. also acknowledge funding from the Japan Society for the Promotion of Science (Grants-in-Aid for Scientific Research (C) Grant No. 17K06173, and JSPS Research Fellow Grant No. 17J00412, respectively). K.Z. and R. J.P. acknowledge funding from the EPSRC (UK) through Grant No. EP/M025187/1. We are grateful to Mr. Kazumi Toda-Peters (OIST) for help with device fabrication using LightFab.

[1] P. S. Virk and H. Baher, The Effect of Polymer Concentration on Drag Reduction, Chem. Eng. Sci. 25, 1183 (1970).

[2] P. S. Virk, H. S. Mickley, and K. A. Smith, Ultimate Asymptote and Mean Flow Structure in Toms Phenomenon, J. Appl. Mech. 37, 488 (1970).

[3] C. M. White and M. G. Mungal, Mechanics and Prediction of Turbulent Drag Reduction with Polymer Additives, Annu. Rev. Fluid Mech. 40, 235 (2008).

[4] D. Samanta, Y. Dubief, M. Holzner, C. Scäfer, A. N. Morozov, C. Wagner, and B. Hof, Elasto-Inertial Turbulence, Proc. Natl. Acad. Sci. U.S.A. 110, 10557 (2013).

[5] Y. Dubief, V. E. Terrapon, and J. Soria, On the Mechanism of Elasto-Inertial Turbulence, Phys. Fluids 25, 110817 (2013).

[6] M. D. Graham, Drag Reduction and the Dynamics of Turbulence in Simple and Complex Fluids, Phys. Fluids 26, 101301 (2014).

[7] D. F. James and J. H. Saringer, Extensional Flow of Dilute Polymer Solutions, J. Fluid Mech. 97, 655 (1980).

[8] M.-N. Wei, B. Li, R. L. A. David, S. C. Jones, V. Sarohia, J. A. Schmitigal, and J. A. Kornfield, Megasupramolecules for Safer, Cleaner Fuel by End Association of Long Telechelic Polymers, Science 350, 72 (2015).

[9] B. Keshavarz, E. C. Houze, J. R. Moore, M. R. Koerner, and G. H. McKinley, Ligament Mediated Fragmentation of Viscoelastic Liquids, Phys. Rev. Lett. 117, 154502 (2016).

[10] S. J. Haward and J. A. Odell, Molecular Orientation in NonNewtonian Flow of Dilute Polymer Solutions around Spheres, Rheol. Acta 43, 350 (2004).

[11] F. J. Galindo-Rosales, L. Campo-Deaño, F. T. Pinho, E. van Bokhorst, P. J. Hamersma, M. S. N. Oliveira, and M. A. Alves, Microfluidic Systems for the Analysis of Viscoelastic Fluid Flow Phenomena in Porous Media, Microfluid. Nanofluid. 12, 485 (2012).

[12] Y. Zhao, A. Q. Shen, and S. J. Haward, Flow of Wormlike Micellar Solutions around Confined Microfluidic Cylinders, Soft Matter 12, 8666 (2016).

[13] A. Varshney and V. Steinberg, Elastic Wake Instabilities in a Creeping Flow between Two Obstacles, Phys. Rev. Fluids 2 , 051301 (2017).

[14] J. Eggers, Nonlinear Dynamics and Breakup of FreeSurface Flows, Rev. Mod. Phys. 69, 865 (1997).
[15] S. L. Anna and G. H. McKinley, Elasto-Capillary Thinning and Breakup of Model Elastic Liquids, J. Rheol. 45, 115 (2001).

[16] L. B. Smolka and A. Belmonte, Drop Pinch-Off and Filament Dynamics of Wormlike Micellar Fluids, J. NonNewtonian Fluid Mech. 115, 1 (2003).

[17] F. Ingremeau and H. Kellay, Stretching Polymers in DropletPinch-Off Experiments, Phys. Rev. X 3, 041002 (2013).

[18] S. J. Haward, R. J. Poole, M. A. Alves, P. J. Oliveira, N. Goldenfeld, and A. Q. Shen, Tricritical Spiral Vortex Instability in Cross-Slot Flow, Phys. Rev. E 93, 031101 (2016).

[19] R. Dylla-Spears, J. E. Townsend, L. Jen-Jacobson, L. L. Sohn, and S. J. Muller, Single-Molecule Sequence Detection via Microfluidic Planar Extensional Flow at a Stagnation Point, Lab Chip 10, 1543 (2010).

[20] J. A. Pathak and S. D. Hudson, Rheo-optics of Equilibrium Polymer Solutions: Wormlike Micelles in Elongational Flow in a Microfluidic Cross-Slot, Macromolecules 39, 8782 (2006).

[21] O. S. Kerr and J. W. Dold, Periodic Steady Vortices in a Stagnation-Point Flow, J. Fluid Mech. 276, 307 (1994).

[22] G. I. Taylor, Production and Dissipation of Vorticity in a Turbulent Fluid, Proc. R. Soc. A 164, 15 (1938).

[23] J.M. Burgers, A Mathematical Model Illustrating the Theory of Turbulence, Adv. Appl. Mech. 1, 171 (1948).

[24] A. C. Robinson and P. G. Saffman, Stability and Structure of Stretched Vortices, Stud. Appl. Math. 70, 163 (1984).

[25] J. C. Neu, The Dynamics of Stretched Vortices, J. Fluid Mech. 143, 253 (1984).

[26] R. G. Larson, E. S. G. Shaqfeh, and S. J. Muller, A Purely Elastic Instability in Taylor-Couette Flow, J. Fluid Mech. 218, 573 (1990).

[27] P. Pakdel and G. H. McKinley, Elastic Instability and Curved Streamlines, Phys. Rev. Lett. 77, 2459 (1996).

[28] G. H. McKinley, P. Pakdel, and A. Öztekin, Rheological and Geometric Scaling of Purely Elastic Flow Instabilities, J. Non-Newtonian Fluid Mech. 67, 19 (1996).

[29] S. J. Haward, G. H. McKinley, and A. Q. Shen, Elastic Instabilities in Planar Elongational Flow of Monodisperse Polymer Solutions, Sci. Rep. 6, 33029 (2016).

[30] O. J. Harris and J. M. Rallison, Instabilities of a Stagnation Point Flow of a Dilute Polymer Solution, J. Non-Newtonian Fluid Mech. 55, 59 (1994).

[31] P. E. Arratia, C. C. Thomas, J. Diorio, and J. P. Gollub, Elastic Instabilities of Polymer Solutions in Cross-Channel Flow, Phys. Rev. Lett. 96, 144502 (2006).

[32] R. J. Poole, M. A. Alves, and P. J. Oliveira, Purely Elastic Flow Asymmetries, Phys. Rev. Lett. 99, 164503 (2007).

[33] L. Xi and M. D. Graham, A Mechanism for Oscillatory Instability in Viscoelastic Cross-Slot Flow, J. Fluid Mech. 622, 145 (2009).

[34] S. J. Haward and G. H. McKinley, Stagnation Point Flow of Wormlike Micellar Solutions in a Microfluidic Cross-Slot Device: Effects of Surfactant Concentration and Ionic Environment, Phys. Rev. E 85, 031502 (2012).

[35] F. A. Cruz, R. J. Poole, A. M. Afonso, F. T. Pinho, P. J. Oliveira, and M. A. Alves, A New Viscoelastic Benchmark Flow: Stationary Bifurcation in a Cross-Slot, J. NonNewtonian Fluid Mech. 214, 57 (2014). 
[36] P. C. Sousa, F. T. Pinho, M. S. N. Oliveira, and M. A. Alves, Purely Elastic Flow Instabilities in Microscale Cross-Slot Devices, Soft Matter 11, 8856 (2015).

[37] A. Groisman and V. Steinberg, Efficient Mixing at Low Reynolds Number Using Polymer Additives, Nature (London) 410, 905 (2001).

[38] A. N. Morozov and W. van Saarloos, An Introductory Essay on Subcritical Instabilities and the Transition to Turbulence in Visco-Elastic Parallel Shear Flows, Phys. Rep. 447, 112 (2007).

[39] L. Pan, A. Morozov, C. Wagner, and P. E. Arratia, NonLinear Elastic Instability in Channel Flows at Low Reynolds Number, Phys. Rev. Lett. 110, 174502 (2013).

[40] B. Qin and P. E. Arratia, Characterizing Elastic Turbulence in Channel Flows at Low Reynolds Number, Phys. Rev. Fluids 2, 083302 (2017).

[41] P. G. De Gennes, Coil-Stretch Transition of Dilute Flexible Polymers under Ultrahigh Velocity Gradients, J. Chem. Phys. 60, 5030 (1974).

[42] R. G. Larson and J. J. Magda, Coil-Stretch Transitions in Mixed Shear and Extensional Flows of Dilute Polymer Solutions, Macromolecules 22, 3004 (1989).

[43] T. T. Perkins, D. E. Smith, and S. Chu, Single Polymer Dynamics in an Elongational Flow, Science 276, 2016 (1997).

[44] D. E. Smith and S. Chu, Response of Flexible Polymers to a Sudden Elongation Flow, Science 281, 1335 (1998).

[45] C. M. Schroeder, H. P. Babcock, E. S. G. Shaqfeh, and S. Chu, Observation of Polymer Conformation Hysteresis in Extensional Flow, Science 301, 1515 (2003).

[46] V. Kantsler, E. Segre, and V. Steinberg, Critical Dynamics of Vesicle Stretching Transition in Elongational Flow, Phys. Rev. Lett. 101, 048101 (2008).

[47] D. R. Gossett, H. T. K. Tse, S. A. Lee, Y. Ying, A. G. Lindgren, O. O. Yang, J. Rao, A. T. Clark, and D. Di Carlo, Hydrodynamic Stretching of Single Cells for Large Population Mechanical Phenotyping, Proc. Natl. Acad. Sci. U.S.A. 109, 7630 (2012).

[48] W. Xu and S. J. Muller, Polymer-Monovalent Salt-Induced DNA Compaction Studied via Single-Molecule Microfluidic Trapping, Lab Chip 12, 647 (2012).

[49] S. J. Haward, M. S. N. Oliveira, M. A. Alves, and G. H. McKinley, Optimized Cross-Slot Geometry for Microfluidic Extensional Rheometry, Phys. Rev. Lett. 109, 128301 (2012).

[50] M. Tanyeri and C.M. Schroeder, Manipulation and Confinement of Single Particles Using Fluid Flow, Nano Lett. 13, 2357 (2013).

[51] C. de Loubens, J. Deschamps, G. Boedec, and M. Leonetti, Stretching of Capsules in an Elongational Flow, A Route to a Constitutive Law, J. Fluid Mech. 767, R3 (2015).

[52] C. Fang, D. Lee, B. Stober, G. G. Fuller, and A. Q. Shen, Integrated Microfluidic Platform for Instantaneous Flow and Localized Temperature Control, RSC Adv. 5, 85620 (2015).

[53] M. Hoffmann, M. Schlüter, and N. Räbiger, Experimental Investigation of Liquid-Liquid Mixing in T-Shaped MicroMixers Using $\mu$-LIF and $\mu$-PIV, Chem. Eng. Sci. 61, 2968 (2006).
[54] A. Fani, S. Camarri, and M. V. Salvetti, Investigation of the Steady Engulfment Regime in a Three-Dimensional T-Mixer, Phys. Fluids 25, 064102 (2013).

[55] D. Vigolo, S. Radl, and H. A. Stone, Unexpected Trapping of Particles at a T-Junction, Proc. Natl. Acad. Sci. U.S.A. 111, 4770 (2014).

[56] J. T. Ault, A. Fani, K. K. Chen, S. Shin, F. Gallaire, and H. A. Stone, Vortex-Breakdown-Induced Particle Capture in Branching Junctions, Phys. Rev. Lett. 117, 084501 (2016).

[57] K. K. Chen, C. W. Rowley, and H. A. Stone, Vortex Breakdown, Linear Global Instability and Sensitivity of Pipe Bifurcation Flows, J. Fluid Mech. 815, 257 (2017).

[58] G. Meineke, M. Hermans, J. Klos, A. Lenenbach, and R. Noll, A Microfluidic Opto-Caloric Switch for Sorting of Particles by Using 3D-Hydrodynamic Focusing Based on SLE Fabrication Capabilities, Lab Chip 16, 820 (2016).

[59] C. D. Meinhart, S. T. Wereley, and M. H. B. Gray, Volume Illumination for Two-Dimensional Particle Image Velocimetry, Meas. Sci. Technol. 11, 809 (2000).

[60] S. T. Wereley and C. D. Meinhart, in Microscale Diagnostic Techniques, edited by K.S. Breuer (Springer-Verlag, Heidelberg, 2005), pp. 51-112.

[61] P. Dontula, C. W. Macosko, and L. Scriven, Model Elastic Liquids with Water-Soluble Polymers, AIChE J. 44, 1247 (1998).

[62] R. B. Bird, R. C. Armstrong, and O. Hassager, Dynamics of Polymeric Liquids (John Wiley and Sons, New York, 1987).

[63] L. Campo-Deaño and C. Clasen, The Slow Retraction Method (SRM) for the Determination of Ultra-Short Relaxation Times in Capillary Breakup Extensional Rheometry Experiments, J. Non-Newtonian Fluid Mech. 165, 1688 (2010).

[64] L. E. Rodd, J. J. Cooper-White, D. V. Boger, and G. H. McKinley, Role of the Elasticity Number in the Entry Flow of Dilute Polymer Solutions in Micro-Fabricated Contraction Geometries, J. Non-Newtonian Fluid Mech. 143, 170 (2007).

[65] J. Brandrup, E. H. Immergut, E. A. Grulke, A. Abe, and D. R. Bloch, Polymer Handbook (Wiley, New York, 1989), Vol. 7.

[66] W. W. Graessley, Polymer Chain Dimensions, and the Dependence of Viscoelastic Properties on Concentration, Molecular Weight and Solvent Power, Polymer 21, 258 (1980).

[67] K. Devanand and J. C. Selser, Asymptotic Behavior and Long-Range Interactions in Aqueous Solutions of Poly (Ethylene Oxide), Macromolecules 24, 5943 (1991).

[68] H. Tadokoro, Structure of Crystalline Polymers (Krieger, Malabar, FL, 1990).

[69] R. J. Poole, The Deborah and Weissenberg Numbers, Rheology Bulletin 53, 32 (2012).

[70] J. H. Ferzinger and M. Perić, Computational Methods for Fluid Dynamics (Springer, Heidelberg, 2002).

[71] M. D. Chilcott and J.M. Rallison, Creeping Flow of Dilute Polymer Solutions Past Cylinders and Spheres, J. Non-Newtonian Fluid Mech. 29, 381 (1988).

[72] P. J. Coates, R. C. Armstrong, and R. A. Brown, Calculation of Steady-State Viscoelastic Flow through Axisymmetrical Contractions with the EEME Formulation, J. NonNewtonian Fluid Mech. 42, 141 (1992). 
[73] P. J. Oliveira, Asymmetric Flows of Viscoelastic Fluids in Symmetric Planar Expansion Geometries, J. NonNewtonian Fluid Mech. 114, 33 (2003).

[74] P. J. Oliveira, F. T. Pinho, and G. A. Pinto, Numerical Simulation of Non-Linear Elastic Flows with a General Collocated Finite-Volume Method, J. Non-Newtonian Fluid Mech. 79, 1 (1998).

[75] P. J. Oliveira, Method for Time-Dependent Simulations of Viscoelastic Flows: Vortex Shedding behind Cylinder, J. Non-Newtonian Fluid Mech. 101, 113 (2001).

[76] M. A. Alves, P. J. Oliveira, and F. T. Pinho, A Convergent and Universally Bounded Interpolation Scheme for the Treatment of Advection, Int. J. Numer. Methods Fluids 41, 47 (2003).

[77] N. Ait Mouheb, D. Malsch, A. Montillet, C. Solliec, and T. Henkel, Numerical and Experimental Investigations of Mixing in T-Shaped and Cross-Shaped Micromixers, Chem. Eng. Sci. 68, 278 (2012).

[78] See Supplemental Material at http://link.aps.org/ supplemental/10.1103/PhysRevX.7.041039 for the following information. Movie M1 depicts the time-dependent elasticity-dominated instability observed by fluorescence microscopy in the $z=0$ plane for the flow of a $0.3 \mathrm{wt} \%$ solution of PEO in water $(\mathrm{El}=10.4)$ flowing at $\mathrm{Re}=15$. Video captured at $20 \mathrm{~Hz}$ using an Andor DSD2 confocal microscope. Movie M2 shows the vorticity field and superimposed streamlines projected in the $x=0$ plane for the flow of water during a quasistatic ramping of the Reynolds number from $\operatorname{Re}=28.3$ up to $\operatorname{Re}=48.3$ and subsequently back to $\operatorname{Re}=23.3$ in increments of $\operatorname{Re}=0.4$. Each frame of the movie is formed from an average of 15 individual vorticity fields captured over 3-s time intervals (effective frame rate $0.33 \mathrm{~Hz}$ ). Movies M3 and M4 depict the timedependent vorticity fields, with superimposed streamlines projected in the $x=0$ plane, for the flow of $0.01 \mathrm{wt} \%$ PEO in water $(\mathrm{El}=0.011)$ at $\mathrm{Re}=19.7$ and $0.03 \mathrm{wt} \% \mathrm{PEO}$ in water $(\mathrm{El}=0.078)$ at $\mathrm{Re}=17.2$, respectively. These movies show instantaneous vorticity fields obtained at a rate of $5 \mathrm{~Hz}$ over time intervals of $3 \mathrm{~s}$.

[79] J. Zilz, C. Schäfer, C. Wagner, R. J. Poole, M. A. Alves, and A. Lindner, Serpentine Channels: Micro-Rheometers for Fluid Relaxation Times, Lab Chip 14, 351 (2014).

[80] R. J. Poole, S. J. Haward, and M. A. Alves, SymmetryBreaking Bifurcations in T-Channel Flows: Effects of Fluid Viscoelasticity, Procedia Eng. 79, 28 (2014).

[81] A. Soleymani, E. Kolehmainen, and I. Turunen, Numerical and Experimental Investigations of Liquid Mixing in T-Type Micromixers, Chem. Eng. J. (Lausanne) 135S, S219 (2008).

[82] J. Soulages and G. H. McKinley, Flow and Stability of Wormlike Micellar and Polymeric Solutions in Converging and T-Shaped Microchannels, AIP Conf. Proc. 1027, 973 (2008).

[83] J. Soulages, M. S. N. Oliveira, P. C. Sousa, M. A. Alves, and G. H. McKinley, Investigating the Stability of Viscoelastic Stagnation Flows in T-Shaped Microchannels, J. NonNewtonian Fluid Mech. 163, 9 (2009).

[84] R. J. Poole, M. Alfateh, and A. P. Gauntlett, Bifurcation in a T-Channel Junction: Effects of Aspect Ratio and ShearThinning, Chem. Eng. Sci. 104, 839 (2013).

[85] A. Aitta, G. Ahlers, and D. S. Cannell, Tricritical Phenomena in Rotating Couette-Taylor Flow, Phys. Rev. Lett. 54, 673 (1985).

[86] A. Aitta, Quantitative Landau Model for Bifurcations Near a Tricritical Point in Couette-Taylor Flow, Phys. Rev. A 34, 2086 (1986).

[87] A. Aitta, Dynamics Near a Tricritical Point in CouetteTaylor Flow, Phys. Rev. Lett. 62, 2116 (1989).

[88] C. S. Dutcher and S. J. Muller, Effects of Weak Elasticity on the Stability of High Reynolds Number Co- and CounterRotating Taylor-Couette Flows, J. Rheol. 55, 1271 (2011).

[89] S. J. Haward and G. H. McKinley, Instabilities in Stagnation Point Flows of Polymer Solutions, Phys. Fluids 25, 083104 (2013).

[90] Y. L. Joo and E. S. G. Shaqfeh, The Effects of Inertia on the Viscoelastic Dean and Taylor-Couette Flow Instabilities with Application to Coating Flows, Phys. Fluids 4, 2415 (1992).

[91] F. A. Cruz, R. J. Poole, A. M. Afonso, F. T. Pinho, P. J. Oliveira, and M. A. Alves, Influence of Channel Aspect Ratio on the Onset of Purely-Elastic Flow Instabilities in Three-Dimensional Planar Cross-Slots, J. Non-Newtonian Fluid Mech. 227, 65 (2016).

[92] K. Kim, C.-F. Li, R. Sureshkumar, S. Balachandar, and R. J. Adrian, Effects of Polymer Stresses on Eddy Structures in Drag-Reduced Turbulent Channel Flow, J. Fluid Mech. 584, 281 (2007).

[93] K. Kim and R. Sureshkumar, Spatiotemporal Evolution of Hairpin Eddies, Reynolds Stress, and Polymer Torque in Polymer Drag-Reduced Turbulent Channel Flows, Phys. Rev. E 87, 063002 (2013).

[94] J. Page and T. A. Zaki, Streak Evolution in Viscoelastic Couette Flow, J. Fluid Mech. 742, 520 (2014).

[95] J. Page and T. A. Zaki, The Dynamics of Spanwise Vorticity Perturbations in Homogeneous Viscoelastic Shear Flow, J. Fluid Mech. 777, 327 (2015).

[96] S. J. Lee and T. A. Zaki, Simulations of Natural Transition in Viscoelastic Channel Flow, J. Fluid Mech. 820, 232 (2017).

[97] J. T. Ault, K. K. Chen, and H. A. Stone, Downstream Decay of Fully Developed Dean Flow, J. Fluid Mech. 777, 219 (2015). 